\title{
PARTIAL EXTENSIONS OF ATTOUCH'S THEOREM WITH APPLICATIONS TO PROTO-DERIVATIVES OF SUBGRADIENT MAPPINGS
}

\author{
ADAM B. LEVY, RENÉ A. POLIQUIN AND LIONEL THIBAULT
}

This paper is dedicated to Professor R. T. Rockafellar on the occasion of his 60 th birthday

\begin{abstract}
Attouch's Theorem, which gives on a reflexive Banach space the equivalence between the Mosco epi-convergence of a sequence of convex functions and the graph convergence of the associated sequence of subgradients, has many important applications in convex optimization. In particular, generalized derivatives have been defined in terms of the epi-convergence or graph convergence of certain difference quotient mappings, and Attouch's Theorem has been used to relate these various generalized derivatives. These relations can then be used to study the stability of the solution mapping associated with a parameterized family of optimization problems. We prove in a Hilbert space several "partial extensions" of Attouch's Theorem to functions more general than convex; these functions are called primal-lower-nice. Furthermore, we use our extensions to derive a relationship between the second-order epi-derivatives of primal-lower-nice functions and the proto-derivative of their associated subgradient mappings.
\end{abstract}

\section{INTRODUCTION}

Various forms of convergence have been studied as means to "differentiate" functions and set-valued mappings (multifunctions). Epi-convergence of a sequence of functions refers to the set-convergence of the sequence of epigraphs of the functions (the epigraph being the set of all points on or above the graph of the function), while the graph convergence of a sequence of set-valued mappings involves the set-convergence of their graphs.

In this paper we study two types of set convergence: Painlevé-Kuratowski and Attouch-Wets convergence. Let $\mathscr{X}$ be a Banach space. Recall that $C_{n} \subset \mathscr{X}$ Painlevé-Kuratowski (PK) converges to $C$, denoted by $C_{n} \rightarrow^{p k} C$ if

$$
\limsup C_{n}=\liminf C_{n}=C ;
$$

Received by the editors February 9, 1994.

1991 Mathematics Subject Classification. Primary 49J52; Secondary 58C20.

Key words and phrases. Attouch's Theorem, epi-derivatives, Mosco epi-convergence, primal lower-nice functions, Attouch-Wets convergence, graph convergence, nonsmooth analysis, sensitivity analysis, optimization, proto-derivatives, Painlevé-Kuratowski convergence, set-valued analysis.

The first author's research was supported in part by the National Science Foundation under grant DMS-9200303. The second author's research was supported in part by the Natural Sciences and Engineering Research Council of Canada under grant OGP41983; it was partly carried out while he was visiting the Université de Pau et des Pays de l'Adour. Thanks to J.P. Penot and L. Thibault. 
for more on PK convergence see [7],[33],[38], and the references therein. Here $\lim \sup C_{n}$ is the set of all accumulation points of sequences from the sets $C_{n}$ and $\lim \inf C_{n}$ is the set of limit points of such sequences. Of course depending on the topology of the space we obtain different kinds of PK convergence. If we do not specify the topology then we assume that the convergence is in the strong topology, and if we are dealing with subsets of the product space $\mathscr{X} \times \mathscr{X}^{*}$ then we assume that the convergence is in the strong-strong topology. (On $\mathscr{X} \times$ $\mathscr{X}^{*}$ we will use the norm $\|(x, u)\|=\max \{\|x\|,\|u\|\}$.) A family of functions $f_{n}: \mathscr{X} \rightarrow \Re \cup\{+\infty\}$ Mosco epi-converges to $f$, denoted by $f_{n} \rightarrow^{m} f$, if epi $f_{n}$ (the epigraph of $f_{n}$ ) PK converges to epi $f$ in both the weak and strong topologies; see [1], and Section 3 of this paper for an alternate description of Mosco epi-convergence.

On the other hand, $C_{n}$ Attouch-Wets (AW) converges to $C$, denoted by $C_{n} \rightarrow^{a w} C$, if for all $\rho$ big enough

$$
\lim _{\operatorname{haus}_{\rho}}\left(C_{n}, C\right) \rightarrow 0,
$$

where

$$
\operatorname{haus}_{\rho}\left(C_{n}, C\right):=\max \left\{e\left(\left(C_{n}\right)_{\rho}, C\right), e\left(C_{\rho}, C_{n}\right)\right\}
$$

where $e(A, B):=\sup _{a \in A} \operatorname{dist}(a, B)$ and $D_{\rho}:=D \cap B_{\rho}$ (where $B_{\rho}$ is the closed ball of radius $\rho$ ). Finally recall that $\left\{f_{n}\right\}$ Attouch-Wets converges to $f$, denoted by $f_{n} \rightarrow^{a w} f$, if epi $f_{n} \rightarrow^{a w}$ epi $f$. For more on Attouch-Wets convergence of sets and functions see [2],[3],[4],[6],[9],[10] and the references therein.

Attouch [1] proved that a sequence of lower semicontinuous proper convex functions on a reflexive Banach space, Mosco epi-converges if and only if the graphs of the subdifferentials Painlevé-Kuratowski converge to the subdifferential of the limit function and a condition that fixes the constant of integration holds. For a family of convex functions several extensions have been given. In a general Banach space Attouch-Beer [5] showed that slice convergence for lower semicontinuous proper convex functions is equivalent to Painlevé-Kuratowski convergence of the graphs of the subdifferentials. A result in the spirit of Attouch's Theorem can also be found in [3] where they show that a family of lower semicontinuous proper convex functions Attouch-Wets converges if and only if the graphs of the subdifferentials Attouch-Wets converge.

Recently, Poliquin [24] extended Attouch's Theorem to possibly nonconvex, primal-lower-nice functions in a finite-dimensional setting. Recall that a lower semicontinuous extended real-valued function $f$ is primal-lower-nice at $\bar{x}$, a point of the effective domain of $f$ (i.e., $\bar{x} \in \operatorname{dom} f:=\{x \mid f(x)<\infty\}$ ), if there exist positive scalars $\bar{\lambda}, \bar{c}$, and $\bar{T}$ such that if $t \geq \bar{T},\|u\| \leq \bar{c} t,\|x-\bar{x}\| \leq \bar{\lambda}$ and $u \in \partial_{p} f(x)$ (the proximal subgradient set to $f$ at $x$; see Section 2) then the inequality

$$
f\left(x^{\prime}\right) \geq f(x)+\left\langle u, x^{\prime}-x\right\rangle-(t / 2)\left\|x^{\prime}-x\right\|^{2}
$$

is valid for all $x^{\prime}$ with $\left\|x^{\prime}-\bar{x}\right\| \leq \bar{\lambda}$. In Proposition 2.2 we give an alternate characterization of primal-lower-nice functions; that characterization says in essence that a function is primal-lower-nice if a portion of the graph of the subdifferential, linearly increasing with $t$, is $t$-monotone. One of the many interesting features of primal-lower-nice functions is that it is not necessary to specify what flavor of subgradients are being employed, as for these functions 
all subgradients are the same. This result is presented in Theorem 2.4, and first appeared in the finite dimensional setting in [23].

Obviously convex functions and lower- $C^{2}$ functions are primal-lower-nice; a function $f$ is lower- $C^{2}$ if $f$ plus a nonnegative multiple of the norm square is convex; see [31]. Poliquin [23] showed that in finite dimensional spaces, the class of primal-lower-nice functions includes the composition of convex functions (not necessarily finite) with $\mathscr{C}^{2}$ mappings and satisfying a constraint qualification. Recently Thibault and Zagrodny [39] showed that in a Banach space, this type of composition function is also primal-lower-nice as long as the convex function is continuous relative to its effective domain. Levy [19] showed that this extra assumption is not needed in a Hilbert space. To be more precise, let $g: \mathscr{X} \rightarrow \Re \cup\{+\infty\}$ be a lower semicontinuous proper convex function, and $F: \mathscr{Y} \rightarrow \mathscr{X}$ be a twice continuously differentiable mapping from the Banach space $\mathscr{Y}$ into the Hilbert space $\mathscr{X}$. If $\bar{x}$ is a point where $g(F(\bar{x}))<\infty$ and the (Robinson) constraint qualification is satisfies at $\bar{x}$, i.e.,

$$
\Re_{+}(\operatorname{dom} g-F(\bar{x}))-\nabla F(\bar{x}) \mathscr{Y}=\mathscr{X},
$$

then $g \circ F$ is primal-lower-nice at $\bar{x}$.

Convexly composite functions are extremely important in analysis and optimization in particular. In fact every lower semicontinuous function on a Banach space that admits a locally uniformly rotund norm can be written as the composition of a lower semicontinuous convex function with a nice mapping; see [28]. However, without extra assumptions it is unlikely that the constraint qualification will be satisfied. A special class of convexly composite functions in finite dimensions that is extremely important in optimization consists of the fully amenable functions. A function is fully amenable if it can be represented as the composition of a piecewise linear-quadratic convex function with a $\mathscr{C}^{2}$ mapping satisfying a constraint qualification. Poliquin and Rockafellar [25]-[27], and Rockafellar [32],[34],[35],[37] have studied fully amenable functions, and have demonstrated their broad applicability to problems in finite-dimensional optimization. Important examples of fully amenable functions include the maximum of finitely many $\mathscr{C}^{2}$ functions, the indicator of a set defined by finitely many $\mathscr{C}^{2}$ constraints for which a constraint qualification is satisfied, and the distance-squared from a convex, polyhedral set to the image of a $\mathscr{C}^{2}$ mapping.

The setting of this paper is that of a Hilbert space. We give three partial extensions of Attouch's Theorem. We show in Theorem 3.5 that if $\left\{f_{n}\right\}$ is a sequence of equi-primal-lower-nice functions at $\bar{x}$ (see Definition 2.1), equibounded below near $\bar{x}$ with $\left\{f_{n}(\bar{x})\right\}$ bounded, then Mosco epi-convergence to $f$ implies that the graphs of the subgradients PK converge to the graph of the subgradients of $f$. Under the same assumptions and a condition that fixes the constant of integration we provide in Theorem 3.6 a partial converse to Theorem 3.5. Indeed we show that if the graphs of the subgradients of $f_{n}$ PK converge to the graph of the subgradients of $f$ (here $f$ is a lower semicontinuous function on $\mathscr{X}$ ), then $f_{n}$ strongly epi-converges to $f$, i.e., convergence of the epigraphs in the strong topology. In is worth noting that a full converse to Theorem 3.5 is not possible, and an example illustrating this is provided in the paragraph before the statement of Theorem 3.6. Finally, again under the same assumptions as Theorem 3.5, we show in Theorem 4.3 that Attouch-Wets convergence of $\left\{f_{n}\right\}$ implies the PK convergence of the graphs of the subgradients, and that we almost 
have Attouch-Wets convergence of the subgradients in the following sense: there exist positive $\alpha$ and $\rho_{0}$ such that for all $\rho \geq \rho_{0}$

$$
e\left(\left(\Gamma^{\alpha}\right)_{\rho}, \Gamma_{2 \alpha}^{n}\right) \rightarrow 0 \text { as } n \rightarrow \infty,
$$

and

$$
e\left(\left(\Gamma_{\alpha}^{n}\right)_{\rho}, \Gamma^{2 \alpha}\right) \rightarrow 0 \quad \text { as } \quad n \rightarrow \infty .
$$

Here

$$
\begin{aligned}
& \Gamma_{\alpha}^{n}=\left\{(x, u) \mid\|x-\bar{x}\|<\alpha, \quad \text { and } \quad u \in \partial f_{n}(x)\right\}, \\
& \Gamma_{2 \alpha}^{n}=\left\{(x, u) \mid\|x-\bar{x}\|<2 \alpha, \quad \text { and } u \in \partial f_{n}(x)\right\}, \\
& \Gamma^{\alpha}=\{(x, u) \mid\|x-\bar{x}\|<\alpha, \quad \text { and } u \in \partial f(x)\}, \\
& \Gamma^{2 \alpha}=\{(x, u) \mid\|x-\bar{x}\|<2 \alpha, \quad \text { and } u \in \partial f(x)\} .
\end{aligned}
$$

Epi-convergence and graph convergence can be applied to various sequences of difference quotients, associated to functions or set-valued mappings, to obtain notions of epi-derivatives of functions and proto-derivatives of set-valued mappings; see [15],[18],[19],[22],[24]-[27],[32], and [34]-[38]. Rockafellar [36] used Attouch's Theorem to show the equivalence between the second-order epidifferentiability of convex functions and the proto-differentiability of their associated subgradient mappings. Poliquin [24] likewise used his extension of Attouch's Theorem to prove the same equivalence but for primal-lower-nice functions on finite-dimensional spaces. Analogously, our partial extensions of Attouch's Theorem enable us to show the following: If $f$ is primal-lower-nice at $\bar{x}$ and $\bar{v} \in \partial f(\bar{x})$, i.e., $\bar{v}$ is a subgradient to $f$ at $\bar{x}$, then

(1) if $f$ is twice Mosco epi-differentiable at $\bar{x}$ relative to $\bar{v}$ then $\partial f$ is PK proto-differentiable at $\bar{x}$ relative to $\bar{v}$; see Theorem 3.8.

(2) If $f$ is twice Attouch-Wets epi-differentiable at $\bar{x}$ relative to $\bar{x}$ then $\partial f$ is Attouch-Wets proto-differentiable at $\bar{x}$ relative to $\bar{v}$; see Theorem 4.4 .

The concepts of second-order epi-derivatives and proto-derivatives are due to Rockafellar; the adjectives Mosco, Attouch-Wets and PK here merely serve to fix the topology (and are not new concepts). Moreover in both cases we obtain a formula for the subgradients of one-half the second order epi-derivatives. In case (1) we obtain that the subgradients of one-half the second-order Mosco epiderivative is equal to the PK proto-derivative of the subgradient mapping; see Theorem 3.8. In case (2) we obtain that the subgradients of the second-order Attouch-Wets epi-derivative is equal to the Attouch-Wets proto-derivative of the subgradient mapping; see Theorem 4.4. Formulas for the second-order epiderivatives are very important in the sensitivity analysis of optimal solutions in parametric optimization where an auxiliary optimization problem can be used to calculate the generalized derivatives; see [25] and [37].

In Zolezzi [40] the following partial extension of Attouch's Theorem is presented: for a family of locally Lipschitzian functions equi lower semidifferentiable and locally equi-bounded on a suitable Banach space, strong epi-convergence implies that

$$
\left(s-w^{*}\right) \lim \sup \operatorname{gph} \partial f_{n} \subset \operatorname{gph} \partial f
$$

(strong convergence in $\mathscr{X}$ and weak $^{*}$ convergence in $\mathscr{X}^{*}$ ). This result is used to show continuous behavior under data perturbations of multipliers and 
generalized gradients associated with integral functionals. In Proposition 3.1 we obtain a similar result for primal-lower-nice functions; we show that if a family of equi-primal-lower-nice functions $\left\{f_{n}\right\}$ strongly epi-converges to $f$ then

$$
(s-w) \lim \sup \operatorname{gph} \partial f_{n} \subset \operatorname{gph} \partial f
$$

(strong convergence in $\mathscr{X}$ and weak convergence in $\mathscr{X}^{*}$ ). Our result in Proposition 3.1 does not follow from Zolezzi's work because primal-lower-nice functions are not in general locally Lipschitzian nor are they in general equi lower semidifferentiable. Finally in Example 3.2 we illustrate that even for convex functions the opposite inclusion in (1.1) does not hold in general, and therefore strong epi-convergence does not necessarily give the convergence of the graphs of the subgradients.

\section{Primal-Lower-Nice functions in Hilbert SPACeS}

In this paper, we are interested in several different kinds of subgradients to functions $f: \mathscr{X} \rightarrow \Re \cup\{+\infty\}:=\Re \cup\{\infty\}$ at points $x \in \mathscr{X}$, where $\mathscr{Z}$ is a Hilbert space. The set of (Clarke) generalized subgradients to $f$ at $x$ is denoted by $\partial f(x)$ (see [13] and [14] for a broad discussion of these subgradients), the set of Fréchet subgradients to $f$ at $x$ is denoted by $\partial_{F} f(x)$ (see [11]), and the set of proximal subgradients to $f$ at $x$, defined below, is denoted by $\partial_{p} f(x)$; for more details on proximal subgradients, see [14] and [30] for instance. A point $u \in \mathscr{X}$ is a proximal subgradient to the function $f$ at $x \in \operatorname{dom} f$, written $u \in \partial_{p} f(x)$, if for some $t>0$ the inequality

$$
f\left(x^{\prime}\right) \geq f(x)+\left\langle u, x^{\prime}-x\right\rangle-(t / 2)\left\|x^{\prime}-x\right\|^{2}
$$

is valid for all $x^{\prime}$ in a neighborhood of $x$. Here $\langle x, y\rangle$ denotes the dot product on $\mathscr{X}$.

The set $\partial_{p} f(x)$ is convex, and the inclusion $\partial_{p} f(x) \subset \partial_{F} f(x) \subset \partial f(x)$ holds in general. For convex functions, the proximal subgradients are the same as the (Clarke) generalized subgradients, and we will see in Theorem 2.4 that this property is an important feature of a more general class of functions, called primal-lower-nice functions.

Definition 2.1. A function $f: \mathscr{X} \rightarrow \Re \cup\{+\infty\}$ is primal-lower-nice at $\bar{x}$ if $f$ is lower semicontinuous, $\bar{x} \in \operatorname{dom} f$ and there exist positive scalars $\bar{\lambda}, \bar{c}$, and $\bar{T}$ such that if $t \geq \bar{T},\|u\| \leq \bar{c} t,\|x-\bar{x}\| \leq \bar{\lambda}$ and $u \in \partial_{p} f(x)$ then the inequality

$$
f\left(x^{\prime}\right) \geq f(x)+\left\langle u, x^{\prime}-x\right\rangle-(t / 2)\left\|x^{\prime}-x\right\|^{2}
$$

is valid for all $x^{\prime}$ with $\left\|x^{\prime}-\bar{x}\right\| \leq \bar{\lambda}$. A family of lower semicontinuous functions is equi-primal-lower-nice at $\bar{x}$ if all the functions in the family are primal-lower-nice at $\bar{x}$ with respect to the same scalars. The function $f$ is called primal-lower-nice if it is primal-lower-nice at all points in its effective domain.

If a function is primal-lower-nice at $\bar{x}$, then we know how steep a quadratic is needed to "realize" any proximal subgradient to $f$ at $\bar{x}$. In particular, convex functions and lower- $C^{2}$ functions are trivially primal-lower-nice. Another important example of a primal-lower-nice function is given by the composition of a lower semicontinuous proper convex function with a twice continuously 
differentiable mapping satisfying a constraint qualification; see [19],[23], and [39].

Primal-lower-nice functions were first introduced in Poliquin [23]. The original definition was in terms of the " $t$-monotonicity" of the proximal subgradient mapping. In finite dimensions, these two ways of characterizing primal-lowernice functions were shown to be equivalent in [23]; we now do the same in the case of a Hilbert space.

We say that a family of functions $f_{n}: \mathscr{X} \rightarrow \Re \cup\{+\infty\}$ is equi-bounded below near $\bar{x}$ if there exists $\lambda$ positive with

$$
\inf _{n \in \mathbb{N}} \inf _{x \in B(\bar{x}, \lambda)}\left\{f_{n}(x)\right\}>-\infty
$$

where $B(y, r)$ denotes the closed ball of radius $r$ centered at $y$.

Proposition 2.2. Let $f_{n}: \mathscr{X} \rightarrow \Re \cup\{+\infty\}$ be a family of lower semicontinuous functions equi-bounded below near $\bar{x}$ and with $\left\{f_{n}(\bar{x})\right\}$ bounded. The following are equivalent :

(1) $\left\{f_{n}\right\}$ is equi-primal-lower-nice at $\bar{x}$.

(2) There exist positive constants $\tilde{\lambda}, \tilde{c}$ and $\widetilde{T}$ such that for all $n \in \mathbb{N}$ and $t \geq \tilde{T}$ we have

$$
\left\langle u_{1}-u_{2}, x_{1}-x_{2}\right\rangle \geq-t\left\|x_{1}-x_{2}\right\|^{2}
$$

whenever $u_{i} \in \partial_{p} f_{n}\left(x_{i}\right),\left\|u_{i}\right\| \leq \tilde{c} t$, and $\left\|x_{i}-\bar{x}\right\| \leq \tilde{\lambda}$.

Proof. We first show that (1) implies (2). Because $\left\{f_{n}\right\}$ is equi-primal-lowernice at $\bar{x}$ there exist positive constants $\bar{c}, \bar{\lambda}$ and $\bar{T}$ such that for all $n \in \mathbb{N}$

$$
f_{n}\left(x^{\prime}\right) \geq f_{n}(x)+\left\langle u, x^{\prime}-x\right\rangle-(t / 2)\left\|x^{\prime}-x\right\|^{2}
$$

is valid for all $x^{\prime}$ with $\left\|x^{\prime}-\bar{x}\right\| \leq \bar{\lambda}$ whenever $t \geq \bar{T},\|u\| \leq \bar{c} t,\|x-\bar{x}\| \leq \bar{\lambda}$ and $u \in \partial_{p} f_{n}(x)$. If $u_{i} \in \partial_{p} f_{n}\left(x_{i}\right),\left\|u_{i}\right\| \leq \bar{c} t, t \geq \bar{T}$ and $\left\|x_{i}-\bar{x}\right\| \leq \bar{\lambda}$, then

$$
\begin{aligned}
& f_{n}\left(x_{1}\right) \geq f_{n}\left(x_{2}\right)+\left\langle u_{2}, x_{1}-x_{2}\right\rangle-(t / 2)\left\|x_{1}-x_{2}\right\|^{2} \text { and } \\
& f_{n}\left(x_{2}\right) \geq f_{n}\left(x_{1}\right)+\left\langle u_{1}, x_{2}-x_{1}\right\rangle-(t / 2)\left\|x_{1}-x_{2}\right\|^{2} .
\end{aligned}
$$

It follows, by adding the previous two inequalities, that

$$
\left\langle u_{1}-u_{2}, x_{1}-x_{2}\right\rangle \geq-t\left\|x_{1}-x_{2}\right\|^{2} .
$$

We now show that (2) implies (1). We may assume without loss of generality that $\left\{f_{n}\right\}$ is equi-bounded below on $B(\bar{x}, \tilde{\lambda})$.

We will need the following claim:

Claim 1. For any $0<\lambda<\tilde{\lambda}$ there exists $T_{1}>0$ such that for all $t \geq T_{1}$, $n \in \mathbb{N}$ and $z \in \mathscr{X}$ with $\|z-\bar{x}\| \leq(\lambda / 4)$ we have

$$
\underset{\|x-\bar{x}\| \leq \lambda}{\operatorname{argmax}}\left\{\langle t z, x\rangle-(t / 2)\|x\|^{2}-f_{n}(x)\right\} \subset B(\bar{x},(3 / 4) \lambda) .
$$

Proof of Claim 1. According to [23, Lemma 3.2], if $\Phi: \mathscr{X} \rightarrow \Re \cup\{+\infty\}$ is lower semicontinuous and bounded below on $B(\bar{x}, \lambda)$ then for

$$
t \geq \frac{2(\Phi(\bar{x})-\gamma)}{(3 / 8) \lambda^{2}}
$$


where $\gamma=\inf _{\|x-x\| \leq \lambda} \Phi(x)$, one has

$$
\underset{\|x-x\| \leq \lambda}{\operatorname{argmax}}\left\{\langle t z, x\rangle-(t / 2)\|x\|^{2}-\Phi(x)\right\} \subset B(\bar{x},(3 / 4) \lambda)
$$

for all $z$ with $\|z-\bar{x}\| \leq(\lambda / 4)$. (Note that [23, Lemma 3.2] is stated in terms of a finite dimensional space, but the only essential requirement is that the norm be given by a dot product.) To complete the proof of the claim just let

$$
T_{1} \geq \sup _{n \in \mathbb{N}} \frac{2\left(f_{n}(\bar{x})-\gamma_{n}\right)}{(3 / 8) \lambda^{2}}
$$

where $\gamma_{n}=\inf _{\|x-x\| \leq \lambda} f_{n}(x)$.

We will also need the following:

Claim 2. There exist positive $\lambda_{2}$ and $T_{2}$ such that for all $n \in \mathbb{N}$ and $t \geq T_{2}$ if $u=t(z-x)$ is in $\partial_{p} f_{n}(x)$ with $\|x-\bar{x}\| \leq\left(\lambda_{2} / 4\right)$ and $\|z-\bar{x}\| \leq\left(\lambda_{2} / 4\right)$ then

$$
f_{n}\left(x^{\prime}\right) \geq f_{n}(x)+\left\langle u, x^{\prime}-x\right\rangle-(t / 2)\left\|x^{\prime}-x\right\|^{2}
$$

for all $x^{\prime} \in B\left(\bar{x}, \lambda_{2}\right)$.

Once the claim has been established just let $\bar{c}=\left(\lambda_{2} / 8\right), \quad \bar{T}=T_{2}$ and $\bar{\lambda}=$ $\left(\lambda_{2} / 8\right)$, then if $u \in \partial_{p} f_{n}(x)$ with $\|u\| \leq \bar{c} t,\|x-\bar{x}\| \leq \bar{\lambda}$ and $t \geq \bar{T}$ then for $z=(u / t)+x$ we have

$$
\|z-\bar{x}\| \leq\|x-\bar{x}\|+\|u\| / t \leq\left(\lambda_{2} / 8\right)+\bar{c} \leq\left(\lambda_{2} / 8\right)+\left(\lambda_{2} / 8\right)=\left(\lambda_{2} / 4\right) .
$$

From Claim 2 it follows that

$$
f_{n}\left(x^{\prime}\right) \geq f_{n}(x)+\left\langle u, x^{\prime}-x\right\rangle-(t / 2)\left\|x^{\prime}-x\right\|^{2}
$$

for all $x^{\prime} \in B(\bar{x}, \bar{\lambda})$.

Proof of Claim 2. Let $0<\lambda_{2}<(1 / 2) \min \{\tilde{\lambda}, \tilde{c}\}$. Let $T_{2}>\max \left\{\tilde{T}, T_{1}\right\}$ where $T_{1}$ is given by Claim 1 with $\lambda=\lambda_{2}$. Fix $n \in \mathbb{N}$, and $t>T_{2}$. Let $u=t(z-x) \in$ $\partial_{p} f_{n}(x)$ with $\|x-\bar{x}\| \leq\left(\lambda_{2} / 4\right)$ and $\|z-\bar{x}\| \leq\left(\lambda_{2} / 4\right)$. Notice that $\|u\|<\tilde{c} t$. Consider the following optimization problem:

$$
\sup _{x^{\prime} \in B\left(x, \lambda_{2}\right)}\left\{\left\langle t z, x^{\prime}\right\rangle-(t / 2)\left\|x^{\prime}\right\|^{2}-f_{n}\left(x^{\prime}\right)\right\} .
$$

(This is a finite number because $f_{n}$ is bounded below on $B\left(\bar{x}, \lambda_{2}\right)$.) Let $\left\{x_{k}\right\}$ be a maximizing sequence, i.e., there exists $\left\{\epsilon_{k}\right\}$ a sequence of nonnegative numbers converging to 0 such that

$$
\left\langle t z, x_{k}\right\rangle-(t / 2)\left\|x_{k}\right\|^{2}-f_{n}\left(x_{k}\right) \geq \sup _{x^{\prime} \in B\left(\bar{x}, \lambda_{2}\right)}\left\{\left\langle t z, x^{\prime}\right\rangle-(t / 2)\left\|x^{\prime}\right\|^{2}-f_{n}\left(x^{\prime}\right)\right\}-\epsilon_{k} .
$$

By Claim 1, we may assume wlog that $\left\{x_{k}\right\} \subset B\left(\bar{x},(3 / 4) \lambda_{2}\right)$. By the BorweinPreiss smooth variational principle (see [12]) there exist $\left\{w_{k}\right\}$, another maximizing sequence, such that

$$
\left\|w_{k}-x_{k}\right\| \leq 2 \sqrt{\epsilon_{k}}
$$

and (eventually)

$$
0 \in \partial_{p}\left[-\langle t z, \cdot\rangle+(t / 2)\|\cdot\|^{2}+f_{n}(\cdot)\right]\left(w_{k}\right)+\sqrt{\epsilon_{k}} B
$$


(where $B$ is the closed unit ball). So there exist $\left\{y_{k}\right\} \in B$ such that

$$
\left(t z-t w_{k}\right)-\sqrt{\epsilon_{k}} y_{k} \in \partial_{p} f_{n}\left(w_{k}\right) .
$$

Eventually $\left\|\left(t z-t w_{k}\right)-\sqrt{\epsilon_{k}} y_{k}\right\|<\tilde{c} t$. To see this notice that

$$
\begin{aligned}
\left\|\left(t z-t w_{k}\right)-\sqrt{\epsilon_{k}} y_{k}\right\| & \leq t\|z-\bar{x}\|+t\left\|\bar{x}-w_{k}\right\|+\sqrt{\epsilon_{k}} \\
& <t\left(\lambda_{2} / 4\right)+t \lambda_{2}+\sqrt{\epsilon_{k}} .
\end{aligned}
$$

Hence eventually $\left\|\left(t z-t w_{k}\right)-\sqrt{\epsilon_{k}} y_{k}\right\|<2 t \lambda_{2}<\tilde{c} t$. Now pick any $\tilde{t}<t$ (not depending on $k)$ such that $\left\|\left(t z-t w_{k}\right)-\sqrt{\epsilon_{k}} y_{k}\right\| \leq \tilde{c} \tilde{t}$, and $\|u\| \leq \tilde{c} \tilde{t}$. We then have

$$
\left\langle\left(t z-t w_{k}\right)-\sqrt{\epsilon_{k}} y_{k}-u, w_{k}-x\right\rangle \geq-\tilde{t}\left\|w_{k}-x\right\|^{2} .
$$

The left-hand side of the previous expression is equal to $-t\left\|w_{k}-x\right\|^{2}-\left\langle\sqrt{\epsilon_{k}} y_{k}\right.$, $\left.w_{k}-x\right\rangle$ (recall that $u=t(z-x)$ ) and therefore we conclude that $\left\{w_{k}\right\}$ converges to $x$. Because $\left\{w_{k}\right\}$ is a maximizing sequence we conclude that the supremum is attained at $x$. (This is because

$$
\left.\limsup _{k \rightarrow \infty}\left\{\left\langle t z, w_{k}\right\rangle-(t / 2)\left\|w_{k}\right\|^{2}-f_{n}\left(w_{k}\right)\right\} \leq\langle t z, x\rangle-(t / 2)\|x\|^{2}-f_{n}(x) .\right)
$$

We have shown that

$$
\left\langle t z, x^{\prime}\right\rangle-(t / 2)\left\|x^{\prime}\right\|^{2}-f_{n}\left(x^{\prime}\right) \leq\langle t z, x\rangle-(t / 2)\|x\|^{2}-f_{n}(x)
$$

for all $x^{\prime} \in B\left(\bar{x}, \lambda_{2}\right)$. From this we conclude that

$$
\begin{aligned}
f_{n}\left(x^{\prime}\right) & \geq\left\langle t z, x^{\prime}-x\right\rangle+(t / 2)\|x\|^{2}-(t / 2)\left\|x^{\prime}\right\|^{2}+f_{n}(x) \\
& =\left\langle t z-t x, x^{\prime}-x\right\rangle-(t / 2)\left\|x^{\prime}-x\right\|^{2}+f_{n}(x),
\end{aligned}
$$

for all $x^{\prime} \in B\left(\bar{x}, \lambda_{2}\right)$. This concludes the proof of Claim 2 and of the proposition.

Remark. The requirement that $\left\{f_{n}\right\}$ be equi-bounded below near $\bar{x}$ and that $\left\{f_{n}(\bar{x})\right\}$ be bounded is only needed to prove that (2) implies (1). Also notice that (1) implies (2) is valid in any Banach space.

Corollary 2.3. Let $f: \mathscr{X} \rightarrow \Re \cup\{+\infty\}$ be a lower semicontinuous function that is finite at $\bar{x}$. The following are equivalent:

(1) $f$ is primal-lower-nice at $\bar{x}$.

(2) There exist positive constants $\tilde{\lambda}, \tilde{c}$ and $\tilde{T}$ such that

$$
\left\langle u_{1}-u_{2}, x_{1}-x_{2}\right\rangle \geq-t\left\|x_{1}-x_{2}\right\|^{2}
$$

whenever $u_{i} \in \partial_{p} f\left(x_{i}\right),\left\|u_{i}\right\| \leq \tilde{c} t, t \geq \tilde{T}$ and $\left\|x_{i}-\bar{x}\right\| \leq \tilde{\lambda}$.

Proof. The function $f$ is bounded below on some neighborhood of $\bar{x}$. (This is because $f$ is lower semicontinuous.) We then apply the previous proposition with $f_{n}=f$ for all $n$.

One of the most important features of primal-lower-nice functions is that their proximal subgradients and (Clarke) generalized subgradients agree. 
Theorem 2.4. If a function $f: \mathscr{X} \rightarrow \Re \cup\{+\infty\}$ is primal-lower-nice at $\bar{x}$, then for all $x$ in a neighborhood of $\bar{x}$, the proximal subgradients to $f$ at $x$ agree with the generalized subgradients to $f$ at $x$, i.e., $\partial_{p} f(x)=\partial f(x)$.

Proof. Because $f$ is primal-lower-nice on a neighborhood of $\bar{x}$ we need only establish the theorem at $\bar{x}$. The proof is similar to Poliquin [23]. Let $\bar{c}>0$, $\bar{\lambda}>0$ and $\bar{T}>0$ be as in the definition of $f$ primal-lower-nice at $\bar{x}$. Since $\mathscr{Z}$ is a reflexive Banach space whose norm is Fréchet differentiable away from zero and locally uniformly convex, Loewen [20] gives the following equality:

$$
\partial f(\bar{x})=\overline{c o}\left[U+U_{0}\right],
$$

where $\overline{c o}$ denotes the closed convex hull, $U$ is the set of weak limit points of sequences of proximal subgradients (i.e. $u \in U$ if there exists a sequence $\left\{x_{n}\right\}$ converging to $\bar{x}$ in norm with $\left\{f\left(x_{n}\right)\right\}$ converging to $f(\bar{x})$, and a sequence $\left\{u_{n}\right\}$ weakly converging to $u$ with $\left.u_{n} \in \partial_{p} f\left(x_{n}\right)\right)$, and $U_{0}$ is the set of singular limit points of sequences of proximal subgradients. (Thus, $u_{0} \in U_{0}$ if and only if there is a sequence $x_{n} \rightarrow \bar{x}$ with $f\left(x_{n}\right) \rightarrow f(\bar{x})$, a sequence $u_{n} \in \partial_{p} f\left(x_{n}\right)$, and a sequence of scalars $\sigma_{n} \rightarrow \infty$ such that $u_{o}$ is the weak limit of $u_{n} / \sigma_{n}$.).

We first show that $\partial_{p} f(\bar{x})$ is a sequentially weakly closed set, by proving that the set $U$ is contained in (and therefore equal to) $\partial_{p} f(\bar{x})$. For $u \in$ $U$, there exist sequences $\left\{x_{n}\right\}$ converging strongly to $\bar{x}$, and $u_{n} \in \partial_{p} f\left(x_{n}\right)$ with $\left\{u_{n}\right\}$ converging weakly to $u$, and $\left\{f\left(x_{n}\right)\right\}$ converging to $f(\bar{x})$. (Notice that the weak convergence of $\left\{u_{n}\right\}$ implies that $\left\|u_{n}\right\|$ is bounded.) Eventually $\left\|x_{n}-\bar{x}\right\| \leq \bar{\lambda}$ and pick $t>\bar{T}$ such that $\left\|u_{n}\right\|<\bar{c} t$. Since $f$ is primal-lower-nice at $\bar{x}$, we eventually have

$$
f(x) \geq f\left(x_{n}\right)+\left\langle u_{n}, x-x_{n}\right\rangle-\frac{t}{2}\left\|x-x_{n}\right\|^{2}
$$

for all $x$ with $\|x-\bar{x}\| \leq \bar{\lambda}$. Taking the limit in (2.2) as $n \rightarrow \infty$, we get $u \in \partial_{p} f(\bar{x})$. Since $\partial_{p} f(\bar{x})$ is convex, its strong closure and sequential weak closure are identical, and we have actually shown that $\partial_{p} f(\bar{x})$ is strongly closed.

Now we consider any $u_{0} \in U_{0}$. It follows that there are sequences $\left\{x_{n}\right\}$ converging strongly to $\bar{x},\left\{\sigma_{n}\right\}$ going to $\infty$, and $u_{n} \in \partial_{p} f\left(x_{n}\right)$ such that $\left\{f\left(x_{n}\right)\right\}$ converges to $f(\bar{x})$ and the sequence of quotients $\left\{u_{n} / \sigma_{n}\right\}$ converges weakly to $u_{0}$. The weak convergence of the quotient sequence implies that there exists a finite number $\gamma$ such that $\left\|u_{n}\right\| \leq \gamma \sigma_{n}$. Since $\left\{x_{n}\right\}$ converges strongly to $\bar{x}$ and $\left\{\sigma_{n}\right\}$ goes to $\infty$, there is an integer $N$ such that the bounds $\left\|x_{n}-\bar{x}\right\| \leq \bar{\lambda}$ and $\gamma \sigma_{n} / \bar{c} \geq \bar{T}$ hold for every $n \geq N$. Since $f$ is primal-lowernice, the inequality

$$
f(x) \geq f\left(x_{n}\right)+\left\langle u_{n}, x-x_{n}\right\rangle-\frac{\gamma \sigma_{n}}{2 \bar{c}}\left\|x-x_{n}\right\|^{2}
$$

is valid for all $x$ with $\|x-\bar{x}\| \leq \bar{\lambda}$. Dividing (2.3) by $\sigma_{n}$ and taking the limit as $n \rightarrow \infty$, we obtain the inequality

$$
0 \geq\left\langle u_{0}, x-\bar{x}\right\rangle-\frac{\gamma}{2 \bar{c}}\|x-\bar{x}\|^{2}
$$

for all $x$ with $\|x-\bar{x}\| \leq \bar{\lambda}$.

Finally, we show that $U+U_{0} \subseteq \partial_{p} f(\bar{x})$ and deduce our result from this. For any $u+u_{0} \in U+U_{0}$, we know from the first part of this proof that $u \in \partial_{p} f(\bar{x})$. 
Therefore, there is a $t>0$ with

$$
f(x) \geq f(\bar{x})+\langle u, x-\bar{x}\rangle-\frac{t}{2}\|x-\bar{x}\|^{2}
$$

locally. This combined with (2.4) yields the inequality

$$
f(x) \geq f(\bar{x})+\left\langle u+u_{0}, x-\bar{x}\right\rangle-\frac{t+\gamma / \bar{c}}{2}\|x-\bar{x}\|^{2}
$$

locally, which implies that that $u+u_{0} \in \partial_{p} f(\bar{x})$. Therefore

$$
\partial f(\bar{x})=\overline{\mathrm{co}}\left[U+U_{0}\right] \subseteq \overline{\mathrm{co}}\left(\partial_{p} f(\bar{x})\right)=\partial_{p} f(\bar{x}) \subseteq \partial f(\bar{x}) .
$$

(The second equality follows because the convex set $\partial_{p} f(\bar{x})$ is strongly closed).

Theorem 2.4 is very useful for studying the properties of the (Clarke) generalized subgradients to primal-lower-nice functions. Our generic approach will be to prove results in terms of the more tractable proximal subgradients, and then state these results in terms of (Clarke) generalized subgradients.

Primal-lower-nice functions have another fascinating property: the "local" Moreau-Yosida approximate of parameter $t$ plus $t / 2$ times the norm square is convex; this was first observed by Thibault and Zagrodny [39]. We denote by $g^{t}$ the Moreau-Yosida approximate of parameter $t$, i.e.,

$$
g^{t}(x)=\inf _{x^{\prime} \in \mathscr{Z}}\left\{g\left(x^{\prime}\right)+(t / 2)\left\|x^{\prime}-x\right\|^{2}\right\} .
$$

Proposition 2.5 [Thibault and Zagrodny]. Let $f_{n}: \mathscr{X} \rightarrow \Re \cup\{+\infty\}$ be a family of lower semicontinuous proper functions, equi-bounded below near $\bar{x}$ with $\left\{f_{n}(\bar{x})\right\}$ bounded. Assume further that $\left\{f_{n}\right\}$ are equi-primal-lower-nice at $\bar{x}$ with constants $\bar{c}, \bar{\lambda}$ and $\bar{T}$. Then there exist $0<c<\bar{c}, T>\bar{T}$ and $0<r_{1}<r_{2}<\bar{\lambda}$ with $r_{1}+c<\bar{\lambda}$ such that for all $t \geq T$ and for all $n$ we have

(1) $\left(f_{n}+\delta_{B\left(\bar{x}, r_{2}\right)}\right)^{t}(x)+(t / 2)\|x\|^{2}$ is convex on $B\left(\bar{x}, r_{1}\right)$ (where $\delta_{C}$ denotes the indicator of the set $C$ ).

(2) The infimum in the definition of $\left(f_{n}+\delta_{B\left(x, r_{2}\right)}\right)^{t}(x)$ is actually equal to the infimum over the set $B\left(\bar{x},(3 / 4) r_{2}\right)$ for each $x$ in $B\left(\bar{x}, r_{1}\right)$.

(3) If $\|u\| \leq c t,\|x-\bar{x}\|<r_{1}$ with $u \in \partial f_{n}(x)$ then

$$
\partial\left(f_{n}+\delta_{B\left(\bar{x}, r_{2}\right)}\right)^{t}\left(x+\frac{u}{t}\right)=\{u\} .
$$

Parts (1) and (2) of the proposition are from [39]. To prove part (3), we will need the following lemma.

Lemma 2.6. Assume

$$
f\left(x^{\prime}\right) \geq f(x)+\left\langle u, x^{\prime}-x\right\rangle-(t / 2)\left\|x^{\prime}-x\right\|^{2}
$$

is valid for all $x^{\prime} \in C$ where $C$ is some set containing $x$. Then $\left(f+\delta_{C}\right)^{t}\left(x+\frac{u}{t}\right)=$ $f(x)+\frac{1}{2 t}\|u\|^{2}$.

Proof. By definition,

$$
\left(f+\delta_{C}\right)^{t}(y)=\inf _{x^{\prime} \in C}\left\{f\left(x^{\prime}\right)+(t / 2)\left\|x^{\prime}-y\right\|^{2}\right\} .
$$


For all $x^{\prime} \in C$ we have

$$
\begin{aligned}
f\left(x^{\prime}\right)+ & (t / 2)\left\|x+\frac{u}{t}-x^{\prime}\right\|^{2} \\
\geq & f(x)+\left\langle u, x^{\prime}-x\right\rangle-(t / 2)\left\|x^{\prime}-x\right\|^{2}+(t / 2)\left\|x-x^{\prime}+\frac{u}{t}\right\|^{2} \\
= & f(x)+\left\langle u, x^{\prime}-x\right\rangle-(t / 2)\left\|x^{\prime}-x\right\|^{2}+(t / 2)\left\|x-x^{\prime}\right\|^{2}+\left\langle x-x^{\prime}, u\right\rangle \\
& +\frac{1}{2 t}\|u\|^{2} \\
= & f(x)+\frac{1}{2 t}\|u\|^{2} .
\end{aligned}
$$

Therefore, $\left(f+\delta_{C}\right)^{t}\left(x+\frac{u}{t}\right)=f(x)+\frac{1}{2 t}\|u\|^{2}$.

Proof of Proposition 2.5. Let $f_{n}$ be equi-primal-lower-nice at $\bar{x}$ with constants $\bar{T}, \bar{\lambda}$ and $\bar{c}$. By [39, Lemmas 4.2 and 4.4] there exist $0<\tilde{r}_{1}<\tilde{r}_{2}$ such that (1) and (2) are valid (with $\tilde{r}_{i}$ in place of $r_{i}$ ). Let $r_{2}=\tilde{r}_{2}$. Pick $0<r_{1}<\tilde{r}_{1}$ and let $0<c<\min \left\{\bar{c},\left(\tilde{r}_{1}-r_{1}\right)\right\}$. We may assume that $T \geq \bar{T}$ and that $r_{2}<\bar{\lambda}$. Fix $n$. Choose any $t \geq T, x$ and $u \in \partial f_{n}(x)$ with $\|x-\bar{x}\|<r_{1}$ and $\|u\| \leq c t$. By the previous lemma and the primal-lower-nice property of $f_{n}$, the infimum in the definition of $\left(f_{n}+\delta_{B\left(\bar{x}, r_{2}\right)}\right)^{t}\left(x+\frac{u}{t}\right)$ is attained at $x$. By [17, Lemma 3.6] we have that

$$
\partial_{F}\left(f_{n}+\delta_{B\left(\bar{x}, r_{2}\right)}\right)^{t}\left(x+\frac{u}{t}\right) \subset \partial_{F} f_{n}(x) \cap\left\{t\left(\left[x+\frac{u}{t}\right]-x\right)\right\} .
$$

In this case, because the function $\left(f_{n}+\delta_{B\left(x, r_{2}\right)}\right)^{t}(x)+(t / 2)\|x\|^{2}$ is convex on $B\left(\bar{x}, \tilde{r}_{1}\right)$, and that $\left\|x+\frac{u}{t}-\bar{x}\right\|<\tilde{r}_{1}$ we have

$\partial_{F}\left(f_{n}+\delta_{B\left(\bar{x}, r_{2}\right)}\right)^{t}\left(x+\frac{u}{t}\right)=\partial_{p}\left(f_{n}+\delta_{B\left(\bar{x}, r_{2}\right)}\right)^{t}\left(x+\frac{u}{t}\right)=\partial\left(f_{n}+\delta_{B\left(\bar{x}, r_{2}\right)}\right)^{t}\left(x+\frac{u}{t}\right)$,

and this set is nonempty. This and (2.5) complete the proof of the Proposition.

\section{MosCo CONVERGENCE}

Recall that $f_{n}: \mathscr{X} \rightarrow \Re \cup\{+\infty\}$ Mosco epi-converges to $f$, denoted by $f_{n} \rightarrow^{m} f$, if $f_{n}$ strongly and weakly epi-converges to $f$. In other words, we have for all $x$

$$
f(x) \leq \liminf f_{n}\left(x_{n}\right) \quad \text { whenever } \quad x_{n} \rightarrow^{w} x
$$

and

$$
\text { there exists } x_{n} \rightarrow^{s} x \text { with } f(x) \geq \lim \sup f_{n}\left(x_{n}\right) .
$$

Note that $f_{n}$ strongly epi-converges to $f$ if the inequality in (3.1) holds for every sequence $x_{n}$ converging strongly to $x$. We will say that $f_{n}$ Mosco epiconverges to $f$ on $C$ if for all $x \in C$

$$
f(x) \leq \liminf f_{n}\left(x_{n}\right) \text { whenever } x_{n} \rightarrow^{w} x \text { and }\left\{x_{n}\right\} \subset C
$$

and

there exists $x_{n} \rightarrow^{s} x$ with $\left\{x_{n}\right\} \subset C$ and $f(x) \geq \lim \sup f_{n}\left(x_{n}\right)$. 
Proposition 3.1. Let $\left\{f_{n}\right\}$ be a family of equi-primal-lower-nice functions with constants $\bar{c}, \bar{\lambda}$ and $\bar{T}$. If $\left\{f_{n}\right\}$ strongly epi-converges to $f$ on $B^{\circ}(\bar{x}, \bar{\lambda})$ (where $C^{\circ}$ is the interior of $\left.C\right)$. then for any $(x, u) \in(s-w) \lim \sup g p h \partial f_{n}$ with $\|x-\bar{x}\|<\bar{\lambda}$ we have $(x, u) \in \operatorname{gph} \partial_{p} f$.

Proof. Let $(x, u) \in(s-w) \lim \sup g p h \partial f_{n}$ with $\|x-\bar{x}\|<\bar{\lambda}$, i.e., there exist subsequences $x_{n_{k}} \rightarrow^{s} x$ and $u_{n_{k}} \rightarrow^{w} u$ with $u_{n_{k}} \in \partial f_{n_{k}}\left(x_{n_{k}}\right)$. For any $x^{\prime}$, with $\left\|x^{\prime}-x\right\|<(\bar{\lambda}-\|x-\bar{x}\|)$, there exist $x_{n}^{\prime} \rightarrow^{s} x^{\prime}$ with $\lim \sup f_{n}\left(x_{n}^{\prime}\right) \leq f\left(x^{\prime}\right)$. Because $\left\{u_{n_{k}}\right\}$ is bounded there exists $t \geq \bar{T}$ with $\left\|u_{n_{k}}\right\| \leq \bar{c} t$. It follows by the primal-lower-nice property that eventually

$$
f_{n_{k}}\left(x_{n_{k}}^{\prime}\right) \geq f_{n_{k}}\left(x_{n_{k}}\right)+\left\langle u_{n_{k}}, x_{n_{k}}^{\prime}-x_{n_{k}}\right\rangle-(t / 2)\left\|x_{n_{k}}^{\prime}-x_{n_{k}}\right\|^{2} .
$$

We then have

$$
\begin{aligned}
f\left(x^{\prime}\right) & \geq \lim \sup f_{n_{k}}\left(x_{n_{k}}^{\prime}\right) \\
& \geq \lim \sup \left\{f_{n_{k}}\left(x_{n_{k}}\right)+\left\langle u_{n_{k}}, x_{n_{k}}^{\prime}-x_{n_{k}}\right\rangle-(t / 2)\left\|x_{n_{k}}^{\prime}-x_{n_{k}}\right\|^{2}\right\} \\
& \geq \liminf \left\{f_{n_{k}}\left(x_{n_{k}}\right)+\left\langle u_{n_{k}}, x_{n_{k}}^{\prime}-x_{n_{k}}\right\rangle-(t / 2)\left\|x_{n_{k}}^{\prime}-x_{n_{k}}\right\|^{2}\right\} \\
& \geq f(x)+\left\langle u, x^{\prime}-x\right\rangle-(t / 2)\left\|x^{\prime}-x\right\|^{2},
\end{aligned}
$$

i.e., $u \in \partial_{p} f(x)$. (The final inequality follows because $\left\{f_{n}\right\}$ strongly epiconverges to $f$.) This completes the proof of the proposition.

We now give an example to illustrate that under the mere assumption of strong epi-convergence there is little hope (in general) that the graphs of the subgradients would converge.

Example 3.2. Consider $l^{2}$ with orthonormal base $\left\{e_{1}, \ldots, e_{n}, \ldots\right\}$. Let $f_{n}$ be a convex function defined by $f_{n}(x):=\delta_{K_{n}}(x)-2\left|\left\langle x, e_{n}\right\rangle\left\langle x, e_{1}\right\rangle\right|^{1 / 2}$ where $K_{n}:=\left\{x \in l^{2} \mid\left\langle x, e_{n}\right\rangle \leq 0,\left\langle x, e_{1}\right\rangle \leq 0\right\}$. Let $f(x):=\delta_{K}(x)$ where $K:=$ $\left\{x \in l^{2} \mid\left\langle x, e_{1}\right\rangle \leq 0\right\}$. Then the sequence of convex functions $f_{n}$ strongly epi-converges to $f$ (but does not Mosco epi-converge). Furthermore, the set of subgradients to $f$ at any $x$ is just the normal cone to the convex set $K$ at the point $x$, and thus in particular $0 \in \partial f(x)$ for any $x \in K$. Fix $x=$ $-\epsilon e_{1} \in K$ with $\epsilon>0$. Notice that the set of subgradients to $f_{n}$ at any point with negative first and $n$th components $-\lambda$ and $-\mu$ is the set $\left\{(\lambda / \mu)^{1 / 2} e_{n}+\right.$ $\left.(\mu / \lambda)^{1 / 2} e_{1}\right\}$, and that there are no subgradients to $f_{n}$ at points with negative first component and a zero $n$th component. Furthermore, any sequence $\left\{x_{n}\right\}$ converging strongly to our fixed $x$ must have $n$th components approaching zero and must eventually have negative first components. Therefore, if there is to be any hope of finding a sequence of subgradients $y_{n} \in \partial f_{n}\left(x_{n}\right)$ converging weakly (or strongly) to $0 \in \partial f(x)$, the $x_{n}$ must eventually have strictly negative first and $n$th components and thus the $y_{n}$ must be of the form $\left(\lambda_{n} / \mu_{n}\right)^{1 / 2} e_{n}+$ $\left(\mu_{n} / \lambda_{n}\right)^{1 / 2} e_{1}$ with $\lambda_{n}$ converging to $\epsilon>0$ and $\mu_{n}$ converging to zero. But such a sequence of $y_{n}$ does not converge weakly (or strongly) to anything, because its norm increases to infinity. We conclude that the graphs of the subgradient mappings $\partial f_{n}$ do not set converge (strong to weak or strong to strong) to the graph of $\partial f$, even though the sequence of functions $f_{n}$ strongly epi-converges to the function $f$.

We will need the following: 
Proposition 3.3 (similar to Attouch-Wets [2, Proposition 4.2]). Let $f_{n}: \mathscr{X} \rightarrow$ $\Re \cup\{+\infty\}$ be a family of functions equi-bounded below near $\bar{x}$ with $\left\{f_{n}(\bar{x})\right\}$ bounded. Assume further that $\left\{f_{n}\right\}$ is equi-primal-lower-nice at $\bar{x}$, with constants $\bar{c}, \bar{\lambda}$ and $\bar{T}$, and that $\left\{f_{n}\right\}$ Mosco epi-converges to $f$ on some neighborhood of $\bar{x}$. Then there exist $T>\bar{T}, 0<c<\bar{c}$ and $0<r_{1}<r_{2}<\bar{\lambda}$ with $c+r_{1}<\bar{\lambda}$ such that all the conclusions of Proposition 2.5 follow and such that for all $t \geq T\left(f_{n}+\delta_{B\left(\bar{x}, r_{2}\right)}\right)^{t}$ Mosco epi-converges to $\left(f+\delta_{B\left(\bar{x}, r_{2}\right)}\right)^{t}$ on $B^{\circ}\left(\bar{x}, r_{1}\right)$. Proof. Let $c>0, T$ and $0<r_{1}<r_{2}$ be given by Proposition 2.5. We may assume that $f_{n}$ Mosco epi-converges to $f$ on $B^{\circ}\left(\bar{x}, r_{2}\right)$. Let $\left(g_{n}\right)^{t}:=$ $\left(f_{n}+\delta_{B\left(\bar{x}, r_{2}\right)}\right)^{t}$ and $g^{t}:=\left(f+\delta_{B\left(\bar{x}, r_{2}\right)}\right)^{t}$. We must show that for all $x$ with $\|x-\bar{x}\|<r_{1}$ and $t \geq T$ we have

$$
g^{t}(x) \leq \liminf \left(g_{n}\right)^{t}\left(x_{n}\right) \quad \text { whenever } \quad x_{n} \rightarrow^{w} x \text { and }\left\|x_{n}-\bar{x}\right\|<r_{1}
$$

and

there exists $x_{n} \rightarrow^{s} x$ with $\left\|x_{n}-\bar{x}\right\|<r_{1}$ and $\quad g^{t}(x) \geq \limsup \left(g_{n}\right)^{t}\left(x_{n}\right)$.

We first show (3.4). For any $\alpha>g^{t}(x)$, there is $x^{\prime}$ with

$$
f\left(x^{\prime}\right)+(t / 2)\left\|x-x^{\prime}\right\|^{2}<\alpha .
$$

By Proposition 2.5 we may assume that $\left\|x^{\prime}-\bar{x}\right\| \leq(3 / 4) r_{2}$. By hypothesis, there exist $x_{n} \rightarrow^{s} x^{\prime}$ with $\lim \sup f_{n}\left(x_{n}\right) \leq f\left(x^{\prime}\right)$. By the definition of $\left(g_{n}\right)^{t}$, eventually we have

$$
\left(g_{n}\right)^{t}(x) \leq\left(f_{n}\right)\left(x_{n}\right)+(t / 2)\left\|x_{n}-x\right\|^{2} .
$$

From this we conclude that $\lim \sup \left(g_{n}\right)^{t}(x)<\alpha$, and we have established (3.4).

To show (3.3) we use a proof similar to [6]. For any sequence $x_{n}$ in the interior of $B\left(\bar{x}, r_{1}\right)$ converging to $x$ weakly, if $\gamma>\liminf \left(g_{n}\right)^{t}\left(x_{n}\right)$ then (by taking a subsequence if necessary) there exist $y_{n}$ such that

$$
f_{n}\left(y_{n}\right)+(t / 2)\left\|x_{n}-y_{n}\right\|^{2}<\gamma .
$$

Again by Proposition 2.5 we may assume that $\left\|y_{n}-\bar{x}\right\| \leq(3 / 4) r_{2}$. By taking a subsequence if necessary, we may assume $x_{n}-y_{n}$ converges weakly in $\mathscr{X}$. It follows thaî $y_{n}$ weakly converges to $y \in \mathscr{X}$ with $\|y-\bar{x}\| \leq(3 / 4) r_{2}$. Therefore

$$
g^{t}(x) \leq f(y)+(t / 2)\|y-x\|^{2} \leq \liminf \left\{f_{n}\left(y_{n}\right)+(t / 2)\left\|x_{n}-y_{n}\right\|^{2}\right\} \leq \gamma .
$$

(The middle inequality holds by the weak-lower semicontinuity of the norm and our assumption that $f_{n}$ Mosco epi-converges to $f$.) By our choice of $\gamma$ we have shown (3.3).

Lemma 3.4. Let $\left\{f_{n}\right\}$ be a sequence of functions from $\mathscr{X}$ into $\Re \cup\{+\infty\}$ that Mosco epi-converges to a function $f: \mathscr{X} \rightarrow \Re \cup\{+\infty\}$ on $B:=B^{\circ}(\bar{x}, r)$. Let $g$ be any continuous convex function from $B$ into $\Re$ that goes to infinity on the boundary of $B$. If we let $g(x)=+\infty$ for $x \notin B$ then $\left\{f_{n}+g\right\}$ Mosco epi-converges to $f+g$ over $\mathscr{X}$.

Proof. We first show that for any $x \in \mathscr{X}$ there exists $\left\{x_{n}\right\}$ strongly converging to $x$ with $\lim \sup \left[f_{n}\left(x_{n}\right)+g\left(x_{n}\right)\right] \leq f(x)+g(x)$. If $x \notin B$ the inequality is obvious. Otherwise, there exists $\left\{x_{n}\right\}$ strongly converging to $x$ with lim sup $f_{n}\left(x_{n}\right) \leq f(x)$. As $g$ is strongly continuous, we have

$\lim \sup \left[f_{n}\left(x_{n}\right)+g\left(x_{n}\right)\right] \leq\left(\lim \sup f_{n}\left(x_{n}\right)\right)+g(x) \leq f(x)+g(x)$. 
Now consider $\left\{x_{n}\right\}$ weakly converging to $x$. Suppose that $\|x-\bar{x}\| \geq r$. As $g$ is weakly lower semicontinuous, we have $+\infty=g(x) \leq \liminf g\left(x_{n}\right)$, and hence

$$
\liminf \left[f_{n}\left(x_{n}\right)+g\left(x_{n}\right)\right] \geq \liminf f_{n}\left(x_{n}\right)+\liminf g\left(x_{n}\right)=+\infty=f(x)+g(x) .
$$

Now suppose that $\|x-\bar{x}\|<r$. If eventually $\left\|x_{n}-\bar{x}\right\| \geq r$, then eventually $g\left(x_{n}\right)=+\infty$ and hence

$$
f(x)+g(x) \leq+\infty=\liminf \left[f_{n}\left(x_{n}\right)+g\left(x_{n}\right)\right] .
$$

So assume that this is not the case. Let

$$
s(0):=\min \left\{n \in \mathbb{N}:\left\|x_{n}-\bar{x}\right\|<r\right\}
$$

and for $k>0$ let

$$
s(k+1):=\min \left\{n \geq 1+s(k):\left\|x_{n}-\bar{x}\right\|<r\right\} .
$$

Let $x_{n}^{\prime}:=x_{s(k)}$ if $n=s(k)$ for some $k \in \mathbb{N}$, and $x_{n}^{\prime}=x$ otherwise. It follows that $x_{n}^{\prime} \in B$ and the sequence weakly converges to $x$. Moreover

$$
\begin{aligned}
\liminf _{n \rightarrow \infty}\left[f_{n}\left(x_{n}\right)\right. & \left.+g\left(x_{n}\right)\right]=\liminf _{k \rightarrow \infty}\left[f_{s(k)}\left(x_{s(k)}\right)+g\left(x_{s(k)}\right)\right] \\
& \geq\left(\liminf _{k \rightarrow \infty} f_{s(k)}\left(x_{s(k)}\right)\right)+g(x) \quad \text { because } g \text { is w-l.s.c. } \\
& \geq \liminf _{n \rightarrow \infty} f_{n}\left(x_{n}^{\prime}\right)+g(x) \quad\left(\left\{x_{s(k)}\right\} \text { is a subsequence of }\left\{x_{n}^{\prime}\right\}\right) \\
& \geq f(x)+g(x),
\end{aligned}
$$

because of the Mosco epi-convergence of $f_{n}$ on $B$. The proof is now complete.

We are now ready to establish our convergence result for Mosco epi-convergence. In the following Theorem we will use the terminology

$$
\operatorname{gph} \partial f_{n} \rightarrow^{p k} \operatorname{gph} \partial f \text { on } B^{\circ}\left(\bar{x}, r_{1}\right) \times \mathscr{X} \text {. }
$$

By this we mean that if $(x, u) \in \lim \operatorname{supgph} \partial f_{n}$ with $\|x-\bar{x}\|<r_{1}$ then $(x, u) \in \liminf \operatorname{gph} \partial f_{n}$ and $(x, u) \in \operatorname{gph} \partial f$ and conversely if $(x, u) \in$ $\operatorname{gph} \partial f$ with $\|x-\bar{x}\|<r_{1}$ then $(x, u) \in \lim \sup \operatorname{gph} \partial f_{n}$.

Theorem 3.5. Let $f_{n}: \mathscr{X} \rightarrow \Re \cup\{+\infty\}$ be a family of functions equi-bounded below near $\bar{x}$ with $\left\{f_{n}(\bar{x})\right\}$ bounded. Assume further that $f_{n}$ are equi-primallower-nice at $\bar{x}$, and that $f_{n}$ Mosco epi-converges to $f$ on some neighborhood of $\bar{x}$. Then $f$ is primal-lower-nice at $\bar{x}$, and there exist $r_{1}$ positive with

$$
\operatorname{gph} \partial f_{n} \rightarrow^{p k} \operatorname{gph} \partial f \text { on } B^{\circ}\left(\bar{x}, r_{1}\right) \times \mathscr{X} \text {. }
$$

Proof. Let $T>0, c>0$ and $0<r_{1}<r_{2}$ be given by Proposition 3.3. We may assume that $r_{1}<(1 / 4) r_{2}$ and that $\left\{f_{n}\right\}$ is equi-bounded below on $B\left(\bar{x}, r_{2}\right)$, and Mosco epi-converges to $f$ on $B^{\circ}\left(\bar{x}, r_{2}\right)$. This ensures that $f$ is also bounded below on $B\left(\bar{x}, r_{2}\right)$.

For all $t \geq T$ we have, by Proposition 3.3, that $\left(f_{n}+\delta_{B\left(x, r_{2}\right)}\right)^{t}$ Mosco epiconverges to $\left(f+\delta_{B\left(\bar{x}, r_{2}\right)}\right)^{t}$ on $B^{\circ}\left(\bar{x}, r_{1}\right)$. Let $\phi$ be a $C^{\infty}$ convex function that goes to infinity on the boundary of $B^{\circ}\left(\bar{x}, r_{1}\right)$. Let $\phi(x):=+\infty$ for $x \notin$ $B^{\circ}\left(\bar{x}, r_{1}\right)$. We have by Lemma 3.4 that

$$
\left(f_{n}+\delta_{B\left(\bar{x}, r_{2}\right)}\right)^{t}+(t / 2)\|\cdot\|^{2}+\phi \rightarrow^{m}\left(f+\delta_{B\left(\bar{x}, r_{2}\right)}\right)^{t}+(t / 2)\|\cdot\|^{2}+\phi \text { on } X
$$


and by Attouch's Theorem

$$
\operatorname{gph}\left(t I+\partial\left(f_{n}+\delta_{B\left(\bar{x}, r_{2}\right)}\right)^{t}+\partial \phi\right) \rightarrow^{p k} \operatorname{gph}\left(t I+\partial\left(f+\delta_{B\left(x, r_{2}\right)}\right)^{t}+\partial \phi\right)
$$

Since $\partial \phi(x)=\nabla \phi(x)$ for $x$ in $B^{\circ}\left(\bar{x}, r_{1}\right)$ and $\partial \phi(x)=\varnothing$ otherwise, we have

$$
\operatorname{gph} \partial\left(f_{n}+\delta_{B\left(x, r_{2}\right)}\right)^{t} \rightarrow^{p k} \operatorname{gph} \partial\left(f+\delta_{B\left(x, r_{2}\right)}\right)^{t}
$$

on $B^{\circ}\left(\bar{x}, r_{1}\right)$.

Let $(x, u) \in \lim \sup g p h \partial f_{n}$ with $\|x-\bar{x}\|<r_{1}$, then, by Proposition 3.1, $(x, u) \in \operatorname{gph} \partial_{p} f$. Therefore

$$
\lim \operatorname{supgph} \partial f_{n} \subset \operatorname{gph} \partial_{p} f \text { over } B\left(\bar{x}, r_{1}\right) \text {. }
$$

Now consider $u \in \partial_{p} f(x)$ with $x \in B\left(\bar{x}, r_{1}\right)$. Because the function $f$ is bounded below, there exists $t>T$ such that $\left\|x+\frac{u}{t}-\bar{x}\right\|<r_{1}$ and such that

$$
f\left(x^{\prime}\right) \geq f(x)+\left\langle u, x^{\prime}-x\right\rangle-(t / 2)\left\|x^{\prime}-x\right\|^{2}
$$

is valid for all $x^{\prime}$ in $B\left(\bar{x}, r_{2}\right)$. It follows by Lemma 2.6 that

$$
\left(f+\delta_{B\left(x, r_{2}\right)}\right)^{t}\left(x+\frac{u}{t}\right)=f(x)+\left(\frac{1}{2 t}\right)\|u\|^{2},
$$

i.e., the infimum is attained at $x$. By [17, Lemma 3.6] we have

$$
\partial\left(f+\delta_{B\left(\bar{x}, r_{2}\right)}\right)^{t}\left(x+\frac{u}{t}\right)=\partial_{F}\left(f+\delta_{B\left(\bar{x}, r_{2}\right)}\right)^{t}\left(x+\frac{u}{t}\right) \subset t\left[x+\frac{u}{t}-x\right] .
$$

(The equality is due to the fact that $\left(f+\delta_{B\left(x, r_{2}\right)}\right)^{t}(\cdot)+(t / 2)\|\cdot\|^{2}$ is convex on $B\left(\bar{x}, r_{1}\right)$.) Therefore $u \in \partial\left(f+\delta_{B\left(x, r_{2}\right)}\right)^{t}\left(x+\frac{u}{t}\right)$. As

$$
\operatorname{gph} \partial\left(f_{n}+\delta_{B\left(x, r_{2}\right)}\right)^{t} \rightarrow^{p k} \operatorname{gph} \partial\left(f+\delta_{B\left(x, r_{2}\right)}\right)^{t}
$$

on $B^{\circ}\left(\bar{x}, r_{1}\right)$, there exist $\left(w_{n}, u_{n}\right)$ with $w_{n} \rightarrow\left(x+\frac{u}{t}\right)$ and $u_{n} \rightarrow u$ with $u_{n} \in \partial\left(f_{n}+\delta_{B\left(x, r_{2}\right)}\right)^{t}\left(w_{n}\right)$. We can write $w_{n}=x_{n}+\frac{u_{n}}{t}$ with $x_{n} \rightarrow x$. Again by [17, Lemma 3.6] we have for $n$ big enough

$$
\begin{aligned}
u_{n} \in & \partial\left(f_{n}+\delta_{B\left(\bar{x}, r_{2}\right)}\right)^{t}\left(x_{n}+\frac{u_{n}}{t}\right)=\partial_{F}\left(f_{n}+\delta_{B\left(\bar{x}, r_{2}\right)}\right)^{t}\left(x_{n}+\frac{u_{n}}{t}\right) \\
& \subset \partial\left(f_{n}+\delta_{B\left(x, r_{2}\right)}\right)\left(x_{n}^{\prime}\right) \cap t\left[x_{n}+\frac{u_{n}}{t}-x_{n}^{\prime}\right],
\end{aligned}
$$

where $x_{n}^{\prime} \in B\left(\bar{x}, r_{2}\right)$ is any point where the infimum in the definition of $\left(f_{n}+\delta_{B\left(x, r_{2}\right)}\right)^{t}\left(x_{n}+\frac{u_{n}}{t}\right)$ is attained. (Such a point exists by the proof of [17, Lemma 3.6].) It follows that $x_{n}^{\prime}=x_{n}$ and that

$$
u_{n} \in \partial\left(f_{n}+\delta_{B\left(x, r_{2}\right)}\right)\left(x_{n}\right)=\partial f_{n}\left(x_{n}\right),
$$

and therefore $(x, u) \in \liminf \operatorname{gph} \partial f_{n}$. We have shown that over $B^{\circ}\left(\bar{x}, r_{1}\right)$

$$
\lim \sup \operatorname{gph} \partial f_{n} \subset \operatorname{gph} \partial_{p} f \subset \liminf \operatorname{gph} \partial f_{n} \text {. }
$$

It follows from Corollary 2.3 that $f$ is primal-lower-nice at $\bar{x}$. This completes the proof of the theorem because $\partial_{p} f=\partial f$ in a neighborhood of $\bar{x}$.

We provide a partial converse to Theorem 3.5. Note that a full converse to Theorem 3.5 cannot hold, as is seen for example by taking the family of functions $\left\{f_{n}\right\}$ given by $f_{n}:=-\|\cdot\|^{2}$ which are (equi) primal-lower-nice at 
0 . In this case, the corresponding subdifferentials are given by $\partial f_{n}(x)=-2 x$. The graphs of these subdifferentials trivially PK converge to the graph of $-2 x$, which is the subdifferential of the function $f=-\|\cdot\|^{2}$. However, the family of functions $\left\{f_{n}\right\}$ does not Mosco epi-converge to $f$ since $-\|\cdot\|^{2}$ is not weakly lower semicontinuous. Notice that in this case $\left\{f_{n}\right\}$ does however strongly epi-converge to $f$.

Theorem 3.6. Let $f_{n}: \mathscr{X} \rightarrow \Re \cup\{+\infty\}$ be a family of functions equi-bounded below near $\bar{x}$ with $\left\{f_{n}(\bar{x})\right\}$ bounded. Assume further that $f_{n}$ are equi-primallower-nice at $\bar{x}$, and that $\operatorname{gph} \partial f_{n} P K$ converges to $\operatorname{gph} \partial f$ on some neighborhood of $\bar{x}$ where $f: \mathscr{X} \rightarrow \Re \cup\{+\infty\}$ is a lower semicontinuous function. Also assume that there exists $\left\{\bar{x}_{n}\right\}$ converging strongly to $\bar{x}$ with $\left\{f\left(\bar{x}_{n}\right)\right\}$ converging to $f(\bar{x})$, and $\left\{\bar{u}_{n}\right\}$ converging weakly to $\bar{u}$ with $\bar{u}_{n} \in \partial f_{n}\left(\bar{x}_{n}\right)$ and $\bar{u} \in \partial f(\bar{x})$. Then $f$ is primal-lower-nice at $\bar{x}$, and $\left\{f_{n}\right\}$ strongly epi-converges to $f$ on a neighborhood of $\bar{x}$.

Proof. By Corollary 2.3 we may assume that $f$ is primal-lower-nice at $\bar{x}$. By Theorem 2.4, $\bar{u}$ is in the proximal subdifferential of $f$ at $\bar{x}$ and hence $f$ is bounded below near $\bar{x}$. Let $T>0, c>0$, and $0<r_{1}<r_{2}$ be given by Proposition 2.5. We may assume that

$f$ is primal-lower-nice at $\bar{x}$ with constants $c, T$ and $r_{2}$. We may further assume that $r_{1}<(1 / 4) r_{2}, \quad\left\{f_{n}\right\} \cup\{f\}$ are equi-bounded below on $B\left(\bar{x}, r_{2}\right)$ and that $\operatorname{gph} \partial f_{n}$ PK converges to $\operatorname{gph} \partial f$ on $B^{\circ}\left(\bar{x}, r_{2}\right)$.

By Lemma 3.7, or by the proof of Theorem 2.64 in Attouch [1] (pages 231232), there exist positive $\rho_{1}^{\prime}<r_{1}$ and $T_{1}^{\prime}>T$ such that for all $t \geq T_{1}^{\prime}$ the functions $\left(f_{n}+\delta_{B\left(\bar{x}, r_{2}\right)}\right)^{t}, \quad n \in \mathbb{N}$, and $\left(f+\delta_{B\left(\bar{x}, r_{2}\right)}\right)^{t}$ are Lipschitzian of constant $c t$ on $B\left(\bar{x}, \rho_{1}^{\prime}\right)$. Let $\gamma:=\min \left\{(1 / 2) c,(1 / 2) \rho_{1}^{\prime}\right\}$. For the same reason there exist positive $\rho_{1}<(1 / 2) \rho_{1}^{\prime}$ and $T_{1}>T_{1}^{\prime}$ such that the above functions are Lipschitzian of constant $\gamma t$ on $B\left(\bar{x}, \rho_{1}\right)$.

Claim. For all $t \geq T_{1}, \operatorname{gph} \partial\left(f_{n}+\delta_{B\left(\bar{x}, r_{2}\right)}\right)^{t}$ PK converges to $\operatorname{gph} \partial\left(f+\delta_{B\left(\bar{x}, r_{2}\right)}\right)^{t}$ on $B^{\circ}\left(\bar{x}, \rho_{1}\right)$.

Proof of Claim. Let $(x, u)$ be an element of $\operatorname{gph} \partial\left(f+\delta_{B\left(x, r_{2}\right)}\right)^{t}$ with $\|x-\bar{x}\|<$ $\rho_{1}$. It follows from Proposition 2.5 part (1) that

$$
u \in \partial_{F}\left(f+\delta_{B\left(\bar{x}, r_{2}\right)}\right)^{t}(x) .
$$

Let $x^{\prime}=x-\frac{u}{t}$. By [17, Lemma 3.6] the infimum in the definition of $\left(f+\delta_{B\left(x, r_{2}\right)}\right)^{t}(x)$ is attained at some $x^{\prime \prime} \in B\left(\bar{x}, r_{2}\right)$, and we have

$$
u \in \partial\left(f+\delta_{B\left(\bar{x}, r_{2}\right)}\right)\left(x^{\prime \prime}\right) \cap\left\{t\left(x^{\prime}+\frac{u}{t}-x^{\prime \prime}\right)\right\} .
$$

It follows that $x^{\prime}=x^{\prime \prime}$. In fact by Proposition 2.5 we have that $x^{\prime}=x^{\prime \prime} \in$ $B\left(\bar{x},(3 / 4) r_{2}\right)$, and therefore we have

$$
u \in \partial\left(f+\delta_{B\left(\bar{x}, r_{2}\right)}\right)\left(x^{\prime}\right)=\partial f\left(x^{\prime}\right) .
$$

By our assumptions there exist $\left(x_{n}^{\prime}, u_{n}\right) \in \operatorname{gph} \partial f_{n}$ converging (strong-strong) to $\left(x^{\prime}, u\right)$ which also means that $\left(x_{n}^{\prime}+\frac{u_{n}}{t}, u_{n}\right)$ converges to $(x, u)$. From (3.5) we have that $\|u\| \leq \gamma t$. (This is because $\left(f+\delta_{B\left(x, r_{2}\right)}\right)^{t}$ is Lipschitzian of constant $\gamma t$.) Therefore

$$
\left\|x^{\prime}-\bar{x}\right\|=\left\|x-\frac{u}{t}-\bar{x}\right\| \leq\|x-\bar{x}\|+\frac{1}{t}\|u\|<\rho_{1}+\gamma \leq \rho_{1}^{\prime} .
$$


Hence eventually $\left\|x_{n}^{\prime}-\bar{x}\right\|<\rho_{1}^{\prime}<r_{1}$, and $\left\|u_{n}\right\| \leq c t$ because $u_{n}$ converges to $u$ and $\|u\| \leq \gamma t<c t$. It follows from the above and Proposition 2.5 part (3) that

$$
\partial\left(f_{n}+\delta_{B\left(x, r_{2}\right)}\right)^{t}\left(x_{n}^{\prime}+\frac{u_{n}}{t}\right)=\left\{u_{n}\right\}
$$

Consequently

$$
(x, u) \in \liminf _{n \rightarrow \infty} \operatorname{gph} \partial\left(f_{n}+\delta_{B\left(\bar{x}, r_{2}\right)}\right)^{t} .
$$

Now let $(x, u) \in \limsup _{n \rightarrow \infty} \operatorname{gph} \partial\left(f_{n}+\delta_{B\left(\bar{x}, r_{2}\right)}\right)^{t}$ with $\|x-\bar{x}\|<\rho_{1}$, we will show that $(x, u) \in \operatorname{gph} \partial\left(f+\delta_{B\left(\bar{x}, r_{2}\right)}\right)^{t}$ and this will conclude the proof of this Claim. By definition there exists a subsequence

$$
\left(x_{n_{k}}, u_{n_{k}}\right) \in \operatorname{gph} \partial\left(f_{n_{k}}+\delta_{B\left(x, r_{2}\right)}\right)^{t}
$$

with $\left(x_{n_{k}}, u_{n_{k}}\right)$ converging to $(x, u)$. By taking another subsequence if necessary we may assume that $\left\|x_{n_{k}}-\bar{x}\right\|<\rho_{1}$. By Proposition 2.5 part (1) we have

$$
u_{n_{k}} \in \partial_{F}\left(f_{n_{k}}+\delta_{B\left(\bar{x}, r_{2}\right)}\right)^{t}\left(x_{n_{k}}\right) .
$$

Let $x_{n_{k}}^{\prime}=x_{n_{k}}-u_{n_{k}} / t$, and $x^{\prime}=x-u / t$. We have $x_{n_{k}}^{\prime}$ converging to $x^{\prime}$ and as in (3.6). (See the discussion prior to (3.6).) It follows that $u_{n_{k}} \in$ $\partial f_{n_{k}}\left(x_{n_{k}}^{\prime}\right)$. By our assumptions $u \in \partial f\left(x^{\prime}\right)$. We also have $\|u\| \leq c t$. (this is because $\left(f_{n_{k}}+\delta_{B\left(\bar{x}, r_{2}\right)}\right)^{t}$ is Lipschitzian of constant $\gamma t$ on $B\left(\bar{x}, \rho_{1}\right)$, which implies that $\left\|u_{n_{k}}\right\| \leq \gamma t$ and in the limit we obtain that $\left.\|u\| \leq \gamma t \leq c t\right)$ and

$$
\left\|x^{\prime}-\bar{x}\right\|=\left\|x-\frac{u}{t}-\bar{x}\right\| \leq\|x-\bar{x}\|+\frac{1}{t}\|u\|<\rho_{1}+\gamma \leq \rho_{1}^{\prime} .
$$

From Proposition 2.5 part (3) we have

$$
\partial\left(f+\delta_{B\left(\bar{x}, r_{2}\right)}\right)^{t}(x)=\partial\left(f+\delta_{B\left(\bar{x}, r_{2}\right)}\right)^{t}\left(x^{\prime}+\frac{u}{t}\right)=\{u\},
$$

and therefore $(x, u) \in \operatorname{gph} \partial\left(f+\delta_{B\left(\bar{x}, r_{2}\right)}\right)^{t}$. This completes the proof of the Claim.

Let $\phi$ be a positive $C^{\infty}$ convex function that goes to infinity at the boundary of $B^{\circ}\left(\bar{x}, \rho_{1}\right)$. We extend $\phi$ to $\mathscr{X}$ by $\phi(x)=+\infty$ for $x$ not in $B^{\circ}\left(\bar{x}, \rho_{1}\right)$. For $t \geq T_{1}$ we have

$$
\begin{aligned}
& \operatorname{gph} \partial[\left.\left(f_{n}+\delta_{B\left(\bar{x}, r_{2}\right)}\right)^{t}+(t / 2)\|\cdot\|^{2}+\phi\right] \\
& \rightarrow^{p k} \operatorname{gph} \partial\left[\left(f+\delta_{B\left(\bar{x}, r_{2}\right)}\right)^{t}+(t / 2)\|\cdot\|^{2}+\phi\right] \text { on } \mathscr{X} .
\end{aligned}
$$

By Proposition 2.5 part (3) once again we may assume (by taking $T_{1}$ bigger if necessary) that for $t \geq T_{1}$ we have $\bar{x}+\frac{\bar{u}}{t}$ and $\bar{x}_{n}+\frac{\bar{u}_{n}}{t}$ in $B^{\circ}\left(\bar{x}, \rho_{1}\right)$,

Hence

$$
\bar{u}_{n} \in \partial\left(f_{n}+\delta_{B\left(\bar{x}, r_{2}\right)}\right)^{t}\left(\bar{x}_{n}+\frac{\bar{u}_{n}}{t}\right) \quad \text { and } \quad \bar{u} \in \partial\left(f+\delta_{B\left(\bar{x}, r_{2}\right)}\right)^{t}\left(\bar{x}+\frac{\bar{u}}{t}\right) .
$$

$\bar{u}_{n}+t\left(\bar{x}_{n}+\frac{\bar{u}_{n}}{t}\right)+\nabla \phi\left(\bar{x}_{n}+\frac{\bar{u}_{n}}{t}\right) \in \partial\left[\left(f_{n}+\delta_{B\left(\bar{x}, r_{2}\right)}\right)^{t}+(t / 2)\|\cdot\|^{2}+\phi\right]\left(\bar{x}_{n}+\frac{\bar{u}_{n}}{t}\right)$,

and

$$
\bar{u}+t\left(\bar{x}+\frac{\bar{u}}{t}\right)+\nabla \phi\left(\bar{x}+\frac{\bar{u}}{t}\right) \in \partial\left[\left(f+\delta_{B\left(\bar{x}, r_{2}\right)}\right)^{t}+(t / 2)\|\cdot\|^{2}+\phi\right]\left(\bar{x}+\frac{\bar{u}}{t}\right) .
$$


Now we apply Attouch's Theorem to deduce that $\left[\left(f_{n}+\delta_{B\left(x, r_{2}\right)}\right)^{t}+(t / 2)\|\cdot\|^{2}+\phi\right]$ Mosco epi-converges to $\left[\left(f+\delta_{B\left(\bar{x}, r_{2}\right)}\right)^{t}+(t / 2)\|\cdot\|^{2}+\phi\right]$ on $\mathscr{X}$ and hence on $B^{\circ}\left(\bar{x}, \rho_{1}\right)$. Finally we apply [1, Theorem 2.65 , p. 232] and [1, Theorem 2.15 (p. 138)] to conclude that $\left\{f_{n}\right\}$ strongly epi-converges to $f$ on $B^{\circ}\left(\bar{x}, \rho_{1}\right)$.

Remark. Instead of the assumption that there exist $\left\{\bar{x}_{n}\right\}$ converging to $\bar{x}$ with $\left\{f\left(\bar{x}_{n}\right)\right\}$ converging to $f(\bar{x})$ and $\left\{\bar{u}_{n}\right\}$ converging weakly to $\bar{u}$ with $\bar{u}_{n}$ in the subdifferential of $f_{n}$ at $\bar{x}_{n}$ and $\bar{u}$ in the subdifferential of $f$ at $\bar{x}$, one might assume that for each ball centered at $\bar{x}$ there is some point $x$ and some sequence $\left\{x_{n}\right\}$ in this ball with $\left\{f\left(x_{n}\right)\right\}$ converging to $f(x)$ and a sequence $\left\{u_{n}\right\}$ converging weakly to some $u$ with $u_{n}$ in the subdifferential of $f_{n}$ at $x_{n}$ and $u$ in the subdifferential of $f$ at $x$.

Lemma 3.7 [1, proof of Theorem 2.64]. Let $\mathscr{F}$ be a family of functions on $\mathscr{Z}$ with values in $\Re \cup\{+\infty\}$ that are equi-bounded below on $\mathscr{X}$ by $-\alpha$ where $\alpha>0$. Assume $\bar{x}$ is a point in $\mathscr{X}$ with $\{f(\bar{x}): f \in \mathscr{F}\}$ bounded above by $\beta>0$. Then for $f \in \mathscr{F}, t>0$ and $x, x^{\prime} \in \mathscr{X}$ we have

$$
f^{t}\left(x^{\prime}\right) \leq f^{t}(x)+(t / 2)\left\|x-x^{\prime}\right\|\left[\left\|x-x^{\prime}\right\|+2\left\{(2 / t)(\beta+\alpha)+\left\|x-x^{\prime}\right\|^{2}\right\}^{1 / 2}\right] .
$$

Proof. Fix $t>0, x, x^{\prime} \in \mathscr{X}$ and $\epsilon>0$. Choose $x_{\epsilon} \in \mathscr{X}$ such that

$$
f\left(x_{\epsilon}\right)+(t / 2)\left\|x-x_{\epsilon}\right\|^{2}<f^{t}(x)+\epsilon
$$

Then

From this it follows that

$$
\begin{aligned}
(t / 2)\left\|x-x_{\epsilon}\right\|^{2} & \leq f^{t}(x)+\epsilon-f\left(x_{\epsilon}\right) \leq f^{t}(x)+\epsilon+\alpha \\
& \leq f(\bar{x})+(t / 2)\|x-\bar{x}\|^{2}+\epsilon+\alpha \\
& \leq \beta+\alpha+\epsilon+(t / 2)\left\|x-x^{\prime}\right\|^{2} .
\end{aligned}
$$

$$
\begin{aligned}
f^{t}\left(x^{\prime}\right) \leq & \leq f\left(x_{\epsilon}\right)+(t / 2)\left\|x_{\epsilon}-x^{\prime}\right\|^{2} \\
& \leq f^{t}(x)+\epsilon-(t / 2)\left\|x_{\epsilon}-x\right\|^{2}+(t / 2)\left\|x_{\epsilon}-x^{\prime}\right\|^{2} \quad(\text { by }(3.7)) \\
& =f^{t}(x)+\epsilon+(t / 2)\left(\left\|x_{\epsilon}-x^{\prime}\right\|-\left\|x_{\epsilon}-x\right\|\right)\left(\left\|x_{\epsilon}-x^{\prime}\right\|+\left\|x_{\epsilon}-x\right\|\right) \\
& \leq f^{t}(x)+\epsilon+(t / 2)\left\|x-x^{\prime}\right\|\left(\left\|x_{\epsilon}-x\right\|+\left\|x_{\epsilon}-x^{\prime}\right\|\right) \\
& \leq f^{t}(x)+\epsilon+(t / 2)\left\|x-x^{\prime}\right\|\left(2\left\|x_{\epsilon}-x\right\|+\left\|x-x^{\prime}\right\|\right) \\
& \leq f^{t}(x)+\epsilon+(t / 2)\left\|x-x^{\prime}\right\|\left[\left\|x-x^{\prime}\right\|\right. \\
& \left.\quad+2\left\{(2 / t)(\beta+\alpha+\epsilon)+\left\|x-x^{\prime}\right\|^{2}\right\}^{1 / 2}\right],
\end{aligned}
$$

and this concludes the proof of the lemma.

For $t>0$ and $\bar{v} \in \partial f(\bar{x})$ define the second-order difference quotients $\phi_{t}$ : $\mathscr{X} \rightarrow \Re \cup\{+\infty\}$ by

$$
\phi_{t}(\xi):=\frac{f(\bar{x}+t \xi)-f(\bar{x})-t\langle v, \xi\rangle}{(1 / 2) t^{2}} .
$$

We say that $f$ is twice Mosco epi-differentiable at $\bar{x}$ relative to $\bar{v}$ if the secondorder difference quotients $\phi_{t}$ Mosco epi-converge as $t \downarrow 0$ to a proper function. The limit function is denoted by $f_{\bar{x}, v}^{\prime \prime} m$. 
Similarly, for a set-valued mapping $\Gamma: \mathscr{X} \rightrightarrows \mathscr{X}$ define the first-order difference quotients $\Gamma_{t}: \mathscr{X} \rightrightarrows \mathscr{X}$ by

$$
\Gamma_{t}(\xi):=\frac{\Gamma(\bar{x}+t \xi)-\bar{v}}{t} .
$$

Let $\bar{v} \in \Gamma(\bar{x})$. We say that $\Gamma$ is Painlevé-Kuratowski (PK) proto-differentiable at $\bar{x}$ relative to $\bar{v}$ with proto-derivative $\Gamma_{\bar{x}, v}^{\prime p k}$ if $\mathrm{gph} \Gamma_{t}$ Painlevé-Kuratowski (PK) converges to $\mathrm{gph} \Gamma_{\dot{x}, v}^{\prime p k}$.

Theorem 3.8. Assume $f$ is primal-lower-nice at $\bar{x}$. If $f$ is twice Mosco epidifferentiable at $\bar{x}$ relative to $\bar{v}$ then $\partial f$ is PK proto-differentiable at $\bar{x}$ relative to $\bar{v}$ with

$$
\partial\left((1 / 2) f_{\bar{x}, v}^{\prime \prime m}\right)(\xi)=(\partial f)_{\bar{x}, v}^{\prime p k}(\xi) .
$$

Proof. Note that for all $t, \quad \phi_{t}(0)=0, \quad \partial\left((1 / 2) \phi_{t}\right)(\xi)=(\partial f(\bar{x}+t \xi)-\bar{v}) / t$ for all $\xi$. ( $\left(\phi_{t}\right.$ is given in (3.8).) By [24], there exist $\rho$ and $t_{0}>0$ such that for all $\left.t \in] 0, t_{0}\right]$ the functions $\phi_{t}$ are equi-bounded below and equi primal-lower-nice on $B(0, \rho)$. We are therefore in a situation where we can use Theorem 3.5. The application of Theorem 3.5 shows that the limiting function $f_{\bar{x}}^{\prime \prime} m$ is primal-lower-nice on the whole space. (This is because it is twice positively homogeneous.) In particular, through Theorem 3.5 we obtain that there exists $r>0$ such that gph $\partial\left((1 / 2) \phi_{t}\right)$ Painlevé-Kuratowski converges to $\operatorname{gph} \partial\left((1 / 2) f_{\bar{x}, v}^{\prime \prime} m\right)$ on $B^{\circ}(0, r) \times \mathscr{X}$. This is sufficient for our purposes because if $\lambda>0$ then $(\xi, \omega) \in \operatorname{gph} \partial\left(f_{\bar{x}, v}^{\prime \prime}\right)$ if and only if $(\lambda \xi, \lambda \omega) \in \operatorname{gph} \partial\left(f_{\bar{x}, 0}^{\prime \prime}\right)$. (Again because $f_{\bar{x}, v}^{\prime \prime}$ is twice positively homogeneous, see Rockafellar [32], and $\partial_{p}\left(f_{x, v}^{\prime \prime m}\right)=\partial\left(f_{x, v}^{\prime \prime}\right)$.) Similarly for any $\lambda>0, \quad(\xi, \omega) \in \lim \sup g p h\left(\partial \phi_{t}\right)$ if and only if $(\lambda \xi, \lambda \omega) \in \lim \sup g p h\left(\partial \phi_{t}\right)$. The same property holds for the liminf and this completes the proof.

\section{Attouch-Wets CONVERGENCE}

Recall that $C_{n}$ Attouch-Wets (AW) converges to $C$, denoted by $C_{n} \rightarrow^{a w} C$, if for all $\rho$ big enough

$$
\lim _{\operatorname{haus}}\left(C_{n}, C\right) \rightarrow 0,
$$

where

$$
\operatorname{haus}_{\rho}\left(C_{n}, C\right)=\max \left\{e\left(\left(C_{n}\right)_{\rho}, C\right), e\left(C_{\rho}, C_{n}\right)\right\}
$$

where $e(A, B):=\sup _{a \in A} \operatorname{dist}(a, B)$ and $D_{\rho}:=D \cap B_{\rho}$ (where $B_{\rho}$ is the closed ball of radius $\rho$ ). Also recall that $\left\{f_{n}\right\}$ Attouch-Wets converges to $f$, denoted by $f_{n} \rightarrow^{a w} f$, if epi $f_{n} \rightarrow^{a w}$ epi $f$.

We will need the following lemmas. For $f, g: \mathscr{X} \rightarrow \Re \cup\{+\infty\}$ let $e_{\rho}(f, g)$ $:=e\left((\text { epi } f)_{\rho}\right.$, epi $\left.g\right)$.

Lemma 4.1. Let $g: \mathscr{X} \rightarrow \Re$ be Lipschitz over any bounded subset of $\mathscr{X}$ and let $f_{n}: \mathscr{X} \rightarrow \Re \cup\{+\infty\}$ and $f: \mathscr{X} \rightarrow \Re \cup\{+\infty\}$ be lower semicontinuous. Assume that for all $\rho \geq \bar{\rho}>0$ we have $\lim _{n \rightarrow \infty}$ haus $_{\rho}$ (epi $f_{n}$, epi $\left.f\right)=0$. Then for all $\rho \geq \bar{\rho}$ there exist $\rho^{\prime} \geq \rho$ and $\lambda>0$ depending only on $g$ and $\rho$ such that 
for all $n$

$$
\begin{aligned}
& e_{\rho}\left(f+g, f_{n}+g\right) \leq 2(1+\lambda) e_{\rho^{\prime}}\left(f, f_{n}\right) \text { and } \\
& e_{\rho}\left(f_{n}+g, f+g\right) \leq 2(1+\lambda) e_{\rho^{\prime}}\left(f_{n}, f\right) .
\end{aligned}
$$

In particular, for all $\rho \geq \bar{\rho}$

$$
\lim _{n \rightarrow \infty} \operatorname{haus}_{\rho}\left(\operatorname{epi}\left(f_{n}+g\right), \operatorname{epi}(f+g)\right)=0 .
$$

Proof. We will only show the first part of (4.1). Consider $(x, r) \in \operatorname{epi}(f+g)_{\rho}$. Then $(x, r-g(x)) \in$ epi $f$ and $|g(x)| \leq|g(0)|+k\|x\| \leq|g(0)|+k \rho$, where $k$ is a Lipschitz constant for $g$ over $B(0, \rho)$. So

$$
|r-g(x)| \leq r+|g(x)| \leq \rho+|g(0)|+k \rho:=\rho^{\prime},
$$

and therefore $(x, r-g(x)) \in(\text { epi } f)_{\rho^{\prime}}$. Take $(u, s) \in$ epi $f_{n}$ with $\|x-u\|+$ $|r-g(x)-s| \leq 2 e_{\rho^{\prime}}\left(f, f_{n}\right)$. But $(u, s+g(u)) \in \operatorname{epi}\left(f_{n}+g\right)$ and

$$
\begin{aligned}
\operatorname{dist}\left((x, r), \operatorname{epi}\left(f_{n}+g\right)\right) & \leq\|(x, r)-(u, s+g(u))\| \\
& \leq\|x-u\|+|r-g(u)-s| \\
& \leq\|x-u\|+|r-g(x)-s|+|g(x)-g(u)| \\
& \leq(1+\lambda)\|x-u\|+|r-g(x)-s| \\
& \leq(1+\lambda)[\|x-u\|+|r-g(x)-s|] \\
& \leq 2(1+\lambda) e_{\rho^{\prime}}\left(f, f_{n}\right),
\end{aligned}
$$

where $\lambda$ is a Lipschitz constant for $g$ over $B(0, \alpha)$, and $\alpha$ is any upper bound for the sequence $\left\{2 e_{\rho^{\prime}}\left(f, f_{n}\right)\right\}$. Therefore

$$
\begin{aligned}
e_{\rho}\left(f+g, f_{n}+g\right) & =\sup _{(x, r) \in \operatorname{epi}(f+g)_{\rho}} \operatorname{dist}\left((x, r), \text { epi }\left(f_{n}+g\right)\right) \\
& \leq 2(1+\lambda) e_{\rho^{\prime}}\left(f, f_{n}\right) .
\end{aligned}
$$

Lemma 4.2. Let $f$ and $f^{\prime}$ be two functions from $\mathscr{X}$ into $\Re$ that are equiLipschitz over each bounded subset of $\mathscr{X}$. Let $\bar{x} \in \mathscr{X}, s>0$ and $\delta:=\delta_{B(\bar{x}, s)}$. Let $\rho>0$ with (epi $f)_{\rho}$, (epi $\left.f^{\prime}\right)_{\rho}$, and (epi $\left.\left(f+f^{\prime}\right)\right)_{\rho}$ nonempty. Finally let $\lambda$ be a Lipschitz constant of $f$ and $f^{\prime}$ over $B\left(\bar{x}, 2 e_{\rho}\left(f, f^{\prime}\right)\right)$. Then

$$
e_{\rho}\left(f+\delta, f^{\prime}+\delta\right) \leq(4+\lambda) e_{\rho}\left(f, f^{\prime}\right) .
$$

Proof. Let $(x, r) \in(\operatorname{epi}(f+\delta))_{\rho}$. Then $|r| \leq \rho$ and $x \in B(\bar{x}, s)$, since $r \geq f(x)+\delta(x)$ and hence $\delta(x)=0$. So $(x, r) \in(\text { epi } f)_{\rho}$ and hence there exists $\left(x^{\prime}, r^{\prime}\right) \in$ epi $f^{\prime}$ with

$$
\left\|x-x^{\prime}\right\| \leq\left\|x-x^{\prime}\right\|+\left|r-r^{\prime}\right| \leq 2 e_{\rho}\left(f, f^{\prime}\right),
$$

and hence

$$
x^{\prime} \in x+B\left(0,2 e_{\rho}\left(f, f^{\prime}\right)\right) \subset \bar{x}+B\left(0, s+2 e_{\rho}\left(f, f^{\prime}\right)\right) .
$$

Choose $x^{\prime \prime} \in B(\bar{x}, s)$ such that $x^{\prime} \in x^{\prime \prime}+B\left(0,2 e_{\rho}\left(f, f^{\prime}\right)\right)$ and put $r^{\prime \prime}=$ $f^{\prime}\left(x^{\prime \prime}\right)+r^{\prime}-f^{\prime}\left(x^{\prime}\right) \geq f^{\prime}\left(x^{\prime \prime}\right)$. We have $\left(x^{\prime \prime}, r^{\prime \prime}\right) \in \operatorname{epi}\left(f^{\prime}+\delta\right)$ since $\left\|x^{\prime \prime}-\bar{x}\right\| \leq s$ 
and furthermore

$$
\begin{aligned}
\operatorname{dist}\left((x, r), \operatorname{epi}\left(f^{\prime}+\delta\right)\right) & \leq\left\|x-x^{\prime \prime}\right\|+\left|r-r^{\prime \prime}\right| \\
& \leq\left\|x-x^{\prime}\right\|+\left|r-r^{\prime}\right|+\left\|x^{\prime}-x^{\prime \prime}\right\|+\left|r^{\prime}-r^{\prime \prime}\right| \\
& =\left\|x-x^{\prime}\right\|+\left|r-r^{\prime}\right|+\left\|x^{\prime}-x^{\prime \prime}\right\|+\left|f^{\prime}\left(x^{\prime}\right)-f^{\prime}\left(x^{\prime \prime}\right)\right| \\
& \leq 4 e_{\rho}\left(f, f^{\prime}\right)+\left|f^{\prime}\left(x^{\prime}\right)-f^{\prime}\left(x^{\prime \prime}\right)\right| \\
& \leq 4 e_{\rho}\left(f, f^{\prime}\right)+\lambda\left\|x^{\prime}-x^{\prime \prime}\right\| \\
& \leq(4+\lambda) e_{\rho}\left(f, f^{\prime}\right)
\end{aligned}
$$

where $\lambda$ is a Lipschitz constant of $f^{\prime}$ over $B\left(\bar{x}, 2 e_{\rho}\left(f, f^{\prime}\right)\right.$ ) (note that $\| x^{\prime}-$ $\left.x^{\prime \prime} \| \leq 2 e_{\rho}\left(f, f^{\prime}\right)\right)$. We conclude that $e_{\rho}\left(f+\delta, f^{\prime}+\delta\right) \leq(4+\lambda) e_{\rho}\left(f, f^{\prime}\right)$.

Theorem 4.3. Let $f_{n}: \mathscr{X} \rightarrow \Re \cup\{+\infty\}$ be a family of functions equi-bounded below on $\mathscr{X}$ with $\left\{f_{n}(\bar{x})\right\}$ bounded. Assume further that $\left\{f_{n}\right\}$ is equi-primallower-nice at $\bar{x}$, and that $\left\{f_{n}\right\}$ Attouch-Wets converges to a lower semicontinuous function $f$. Then $f$ is primal-lower-nice at $\bar{x}$. In addition there exist positive $\alpha$ and $\rho_{0}$ such that

$$
\operatorname{gph} \partial f_{n} \rightarrow^{p k} \operatorname{gph} \partial f \text { on } B^{\circ}(\bar{x}, \alpha) \times \mathscr{X},
$$

and for all $\rho \geq \rho_{0}$

$$
e\left(\left(\Gamma^{\alpha}\right)_{\rho}, \Gamma_{2 \alpha}^{n}\right) \rightarrow 0 \text { as } n \rightarrow \infty
$$

and

$$
e\left(\left(\Gamma_{\alpha}^{n}\right)_{\rho}, \Gamma^{2 \alpha}\right) \rightarrow 0 \text { as } n \rightarrow \infty
$$

Here

$$
\begin{aligned}
& \Gamma_{\alpha}^{n}=\left\{(x, u) \mid\|x-\bar{x}\|<\alpha, \quad \text { and } u \in \partial f_{n}(x)\right\}, \\
& \Gamma_{2 \alpha}^{n}=\left\{(x, u) \mid\|x-\bar{x}\|<2 \alpha, \quad \text { and } u \in \partial f_{n}(x)\right\}, \\
& \Gamma^{\alpha}=\{(x, u) \mid\|x-\bar{x}\|<\alpha, \quad \text { and } u \in \partial f(x)\}, \\
& \Gamma^{2 \alpha}=\{(x, u) \mid\|x-\bar{x}\|<2 \alpha, \quad \text { and } u \in \partial f(x)\} .
\end{aligned}
$$

Proof. First note that $f$ is also bounded below on $\mathscr{X}$. Assume that $f_{n}$ are equi-primal-lower-nice with constants $\bar{\lambda}, \bar{c}$ and $\bar{T} \geq 2$. Let $T, c, r_{1}, r_{2}$ be given by Proposition 2.5. By taking $T$ bigger if necessary we may assume that for all $x$ with $\|x-\bar{x}\|<r_{1}$ and $t \geq T$

$\left(f_{n}\right)^{t}(x):=\inf _{x^{\prime}}\left\{f_{n}\left(x^{\prime}\right)+(t / 2)\left\|x^{\prime}-x\right\|^{2}\right\}=\inf _{\left\|x^{\prime}-\bar{x}\right\| \leq r_{2}}\left\{f_{n}\left(x^{\prime}\right)+(t / 2)\left\|x^{\prime}-x\right\|^{2}\right\}$,

and that the same is true for $f$. In other words for all $t \geq T$ and $x \in B^{\circ}\left(\bar{x}, r_{1}\right)$ we have $\left(f_{n}\right)^{t}(x)=\left(f_{n}+\delta_{B\left(\bar{x}, r_{2}\right)}\right)^{t}(x)$ and $f^{t}(x)=\left(f+\delta_{B\left(\bar{x}, r_{2}\right)}\right)^{t}(x)$.

Take $r_{0}$ positive such that $r_{0}<r_{1}$. For all $t \geq T$ we have by [6, Lemma 3.3] (by taking $T$ bigger if necessary) that

$$
\left(f_{n}\right)^{t} \rightarrow^{a w} f^{t}
$$

To be more precise, because $\left\{f_{n}, f\right\}$ is equi-bounded below, we have, again by [6, Lemma 3.3], that there exists $\bar{\rho}$ such that for $\rho \geq \bar{\rho}$ and $t \geq T$ we have

$$
\lim _{n \rightarrow \infty} \operatorname{haus}_{\rho}\left(\operatorname{epi}\left(f_{n}\right)^{t} \text {, epi } f^{t}\right)=0 \text {. }
$$


From Lemma 4.1 it follows that

$$
\lim _{n \rightarrow \infty} \operatorname{haus}_{\rho}\left(\text { epi }\left[\left(f_{n}\right)^{t}+(t / 2)\|\cdot\|^{2}\right] \text {, epi }\left[f^{t}+(t / 2)\|\cdot\|^{2}\right]\right)=0 \text {. }
$$

From Lemma 4.2 we have for $\rho \geq \bar{\rho}$ and $t \geq T$

$\lim _{n \rightarrow \infty} \operatorname{haus}_{\rho}\left(\operatorname{epi}\left[\left(f_{n}\right)^{t}+(t / 2)\|\cdot\|^{2}+\delta_{B\left(\bar{x}, r_{0}\right)}\right]\right.$, epi $\left.\left[f^{t}+(t / 2)\|\cdot\|^{2}+\delta_{B\left(\bar{x}, r_{0}\right)}\right]\right)=0$.

From [6, Theorem 5.2] (see also [3, Theorem 2.3] and [21, Theorem 3.6]) we obtain that for $\rho \geq \bar{\rho}$ and $t \geq T$

$$
\lim _{n \rightarrow \infty} \operatorname{haus}_{\rho}\left(\operatorname{gph}\left[t I+\partial\left(\left(f_{n}\right)^{t}+\delta_{B\left(\bar{x}, r_{0}\right)}\right)\right], \operatorname{gph}\left[t I+\partial\left(f^{t}+\delta_{B\left(\bar{x}, r_{0}\right)}\right)\right]\right)=0,
$$

and from this we deduce that

$$
\lim _{n \rightarrow \infty} \operatorname{haus}_{\rho}\left(\operatorname{gph} \partial\left(\left(f_{n}\right)^{t}+\delta_{B\left(\bar{x}, r_{0}\right)}\right), \operatorname{gph} \partial\left(f^{t}+\delta_{B\left(\bar{x}, r_{0}\right)}\right)\right)=0 .
$$

Notice that we also have

$$
\begin{array}{rl}
f_{n} \rightarrow^{a w} & f \Longrightarrow\left[\left(f_{n}\right)^{t}+(t / 2)\|\cdot\|^{2}+\delta_{B\left(\bar{x}, r_{0}\right)}\right] \rightarrow^{m}\left[f^{t}+(t / 2)\|\cdot\|^{2}+\delta_{B\left(\bar{x}, r_{0}\right)}\right] \\
& \Longrightarrow \operatorname{gph}\left[t I+\partial\left(\left(f_{n}\right)^{t}+\delta_{B\left(\bar{x}, r_{0}\right)}\right)\right] \rightarrow^{p k} \operatorname{gph}\left[t I+\partial\left(f^{t}+\delta_{B\left(\bar{x}, r_{0}\right)}\right)\right] \\
& \Longrightarrow \operatorname{gph} \partial\left(\left(f_{n}\right)^{t}+\delta_{B\left(\bar{x}, r_{0}\right)}\right) \rightarrow^{p k} \operatorname{gph} \partial\left(f^{t}+\delta_{B\left(\bar{x}, r_{0}\right)}\right) .
\end{array}
$$

Where the implication in (4.9) is due to the fact that for convex functions, Attouch-Wets convergence implies Mosco epi-convergence; see [6, Proposition 4.5]. The implication in (4.10) follows of course by Attouch's Theorem. Because of these facts we may proceed as in the proof of Theorem 3.5 to obtain that the graph of the subdifferential of $f_{n}$ PK converges to the graph of the subdifferential of $f$ over some neighborhood of $\bar{x}$. It then follows by Corollary 2.3 that $f$ is primal-lower-nice at $\bar{x}$ and that the Mosco epi-convergence assertion holds as in Theorem 3.5. We may now assume that $f$ is primal-lower-nice at $\bar{x}$ with constants $T, c$, and $3 \alpha$, with $3 \alpha<r_{0}$.

We first show (4.3). Fix $\rho>\bar{\rho}$ and $x$ satisfying $\|x\| \leq \rho$. Also fix $t>T$ (independent of $x$ ) with $\rho<t \min \{c, \alpha\}$. Assume $u \in \partial f(x)$ with $\|u\| \leq$ $\rho$. (Note that $\rho$ is independent of $x$.) By (4.5) and Lemma 2.6, note that $\left\|x+\frac{u}{t}-\bar{x}\right\|<2 \alpha$, the infimum in the definition of $f^{t}$ at $\left(x+\frac{u}{t}\right)$ is attained at $x$. It follows that $u \in \partial f^{t}\left(x+\frac{u}{t}\right)$. Let $\rho_{t}:=\rho(1+(1 / t))$. We have that $\left\|x+\frac{u}{t}\right\| \leq \rho_{t}$ and that $\|u\| \leq \rho_{t}$. Let

$$
\gamma_{n}:=\operatorname{haus}_{\rho_{t}}\left(\operatorname{gph} \partial\left(\left(f_{n}\right)^{t}+\delta_{B\left(\bar{x}, r_{0}\right)}\right), \operatorname{gph} \partial\left(f^{t}+\delta_{B\left(\bar{x}, r_{0}\right)}\right)\right) .
$$

(note that $\gamma_{n} \rightarrow 0$ as $n \rightarrow \infty$.) Choose $n$ big enough so that $\gamma_{n}<(1 / 4) \alpha$. Consider any $\epsilon$ positive with $\epsilon<(1 / 4) \alpha$. There exist $x_{\epsilon}$ and $u_{\epsilon}$ such that $u_{\epsilon} \in \partial\left(\left(f_{n}\right)^{t}+\delta_{B\left(x, r_{0}\right)}\right)\left(x_{\epsilon}+\frac{u_{\epsilon}}{t}\right)$ with

$$
\left\|\left(x_{\epsilon}+\frac{u_{\epsilon}}{t}\right)-\left(x+\frac{u}{t}\right)\right\|-\epsilon \leq \gamma_{n}, \quad\left\|u_{\epsilon}-u\right\|-\epsilon \leq \gamma_{n} .
$$

This is because

$$
\begin{aligned}
\gamma_{n} & \geq e\left(\operatorname{gph} \partial\left(f^{t}+\delta_{B\left(\bar{x}, r_{0}\right)}\right)_{\rho_{t}}, \operatorname{gph} \partial\left(\left(f_{n}\right)^{t}+\delta_{B\left(\bar{x}, r_{0}\right)}\right)\right) \\
& \geq \operatorname{dist}\left(\left(x+\frac{u}{t}, u\right), \operatorname{gph} \partial\left(\left(f_{n}\right)^{t}+\delta_{B\left(\bar{x}, r_{0}\right)}\right)\right) .
\end{aligned}
$$


Notice that $\left\|x_{\epsilon}+\frac{u_{\epsilon}}{t}-\bar{x}\right\|<3 \alpha$, which implies that $u_{\epsilon} \in \partial\left(f_{n}\right)^{t}\left(x_{\epsilon}+\frac{u_{\epsilon}}{t}\right)$ and hence by [17, Lemma 3.6] (see the proof of Theorem 3.5) that $u_{\epsilon} \in \partial f_{n}\left(x_{\epsilon}\right)$. We have

$$
\begin{aligned}
\left\|x_{\epsilon}-x\right\| & \leq(1 / t)\left\|u_{\epsilon}-u\right\|+\gamma_{n}+\epsilon \\
& \leq(1+(1 / t)) \gamma_{n}+(1+(1 / t)) \epsilon .
\end{aligned}
$$

Also we have

$$
\left\|u_{\epsilon}-u\right\| \leq(1+(1 / t)) \gamma_{n}+\epsilon .
$$

It follows by the choice of $n$ and $\epsilon$ that $\left\|x_{\epsilon}-\bar{x}\right\|<2 \alpha$, and

$$
\begin{aligned}
& \operatorname{dist}\left((u, x), \Gamma_{2 \alpha}^{n}\right) \leq \max \left\{\left\|u_{\epsilon}-u\right\|,\left\|x_{\epsilon}-x\right\|\right\} \\
& \leq(1+(1 / t)) \gamma_{n}+(1+(1 / t)) \epsilon .
\end{aligned}
$$

This shows that

$$
\operatorname{dist}\left((u, x), \Gamma_{2 \alpha}^{n}\right) \leq(1+(1 / t)) \gamma_{n},
$$

and hence

$$
e\left(\left(\Gamma^{\alpha}\right)_{\rho}, \Gamma_{2 \alpha}^{n}\right) \leq(1+(1 / t)) \gamma_{n} .
$$

By taking $n \rightarrow \infty$ we have

$$
e\left(\left(\Gamma^{\alpha}\right)_{\rho}, \Gamma_{2 \alpha}^{n}\right) \rightarrow 0 \text { as } n \rightarrow \infty .
$$

To show (4.4), fix $\delta>0$ and choose $N \in \mathbb{N}$ such that for all $n \geq N$ we have $\gamma_{n} \leq \min \{(1 / 4) \alpha,(\delta / 2)\}$. (Here $\gamma_{n}$ is defined in (4.11).) Consider for any fixed $n \geq N, u_{n} \in \partial f_{n}\left(x_{n}\right)$ with $\left\|x_{n}\right\| \leq \rho,\left\|x_{n}-\bar{x}\right\|<\alpha$ and $\left\|u_{n}\right\| \leq \rho$, where $\rho \geq \bar{\rho}$. Fix $t \geq T$ satisfying $\rho \leq t \min \{c, \alpha\}$. By Proposition 2.5 we have $u_{n} \in \partial\left(f_{n}\right)^{t}\left(x_{n}+\frac{u_{n}}{t}\right)$. (This is because $\left\|x_{n}+\frac{u_{n}}{t}-\bar{x}\right\|<2 \alpha$.) Again let $\rho_{t}=\rho(1+(1 / t))$. Fix $\delta>0$. Fix $\epsilon$ positive with $\epsilon<(1 / 4) \alpha$. There exist $x_{\epsilon}$ and $u_{\epsilon}$ such that $u_{\epsilon} \in \partial\left(f^{t}+\delta_{B\left(x, r_{0}\right)}\right)\left(x_{\epsilon}+\frac{u_{\epsilon}}{t}\right)$ with

$$
\left\|\left(x_{\epsilon}+\frac{u_{\epsilon}}{t}\right)-\left(x_{n}+\frac{u_{n}}{t}\right)\right\|-\epsilon \leq \gamma_{n}, \quad\left\|u_{\epsilon}-u_{n}\right\|-\epsilon \leq \gamma_{n} .
$$

This is because

$$
\begin{aligned}
\gamma_{n} & \geq e\left(\operatorname{gph} \partial\left(\left(f_{n}\right)^{t}+\delta_{B\left(\bar{x}, r_{0}\right)}\right)_{\rho_{t}}, \operatorname{gph} \partial\left(f^{t}+\delta_{B\left(\bar{x}, r_{0}\right)}\right)\right) \\
& \geq \operatorname{dist}\left(\left(x_{n}+\frac{u_{n}}{t}, u_{n}\right), \operatorname{gph} \partial\left(f^{t}+\delta_{B\left(\bar{x}, r_{0}\right)}\right)\right) .
\end{aligned}
$$

Notice that $\left\|x_{\epsilon}+\frac{u_{\epsilon}}{t}-\bar{x}\right\|<3 \alpha$, which implies that $u_{\epsilon} \in \partial\left(f_{n}\right)^{t}\left(x_{\epsilon}+\frac{u_{\epsilon}}{t}\right)$ and hence by [17, Lemma 3.6] (see the proof of Theorem 3.5) that $u_{\epsilon} \in \partial f\left(x_{\epsilon}\right)$. We have

$$
\begin{aligned}
\left\|x_{\epsilon}-x_{n}\right\| & \leq(1 / t)\left\|u_{\epsilon}-u_{n}\right\|+\gamma_{n}+\epsilon \\
& \leq(1+(1 / t)) \gamma_{n}+(1+(1 / t)) \epsilon .
\end{aligned}
$$

Also we have

$$
\left\|u_{\epsilon}-u_{n}\right\| \leq(1+(1 / t)) \gamma_{n}+\epsilon .
$$

It follows by the choice of $n$ and $\epsilon$ that $\left\|x_{\epsilon}-\bar{x}\right\|<2 \alpha$. Then

$$
\begin{aligned}
& \operatorname{dist}\left(\left(u_{n}, x_{n}\right), \Gamma^{2 \alpha}\right) \leq \max \left\{\left\|u_{\epsilon}-u\right\|,\left\|x_{\epsilon}-x\right\|\right\} \\
& \quad \leq(1+(1 / t)) \gamma_{n}+(1+(1 / t)) \epsilon .
\end{aligned}
$$

This shows that

$$
\operatorname{dist}\left(\left(u_{n}, x_{n}\right), \Gamma^{2 \alpha}\right) \leq(1+(1 / t)) \gamma_{n}
$$


Thus we have that for all $n \geq N$

$$
e\left(\left(\Gamma_{\alpha}^{n}\right)_{\rho}, \Gamma^{2 \alpha}\right) \leq 2 \gamma_{n} \leq \delta .
$$

From this we conclude that

$$
e\left(\left(\Gamma_{\alpha}^{n}\right)_{\rho}, \Gamma^{2 \alpha}\right) \rightarrow 0 \text { as } n \rightarrow \infty .
$$

This completes the proof of the theorem.

For $\bar{v} \in \partial f(\bar{x})$ we say that $f$ is twice Attouch-Wets epi-differentiable at $\bar{x}$ relative to $\bar{v}$ if the second order difference quotients (see (3.8)) Attouch-Wets converge as $t \downarrow 0$ to a proper function. The limiting function (which we may assume is closed) is denoted by $f_{\bar{x}, \bar{v}}^{\prime \prime}$. It is easy to show that the function $f_{\bar{x}, \bar{v}}^{\prime \prime}$ is twice positively homogeneous.

For a set-valued mapping $\Gamma: \mathscr{X} \rightarrow \mathscr{X}$ we say that $\Gamma$ is Attouch-Wets protodifferentiable at $\bar{x}$ relative to $\bar{v}$ where $\bar{v} \in \Gamma(\bar{x})$ if the first-order difference quotients (see (3.9)) Attouch-Wets converge to $\mathrm{gph} \Gamma_{\bar{x}, v}^{\prime a w}$.

Theorem 4.4. Assume $f$ is primal-lower-nice at $\bar{x}$. If $f$ is twice Attouch-Wets epi-differentiable at $\bar{x}$ relative to $\bar{v}$ then $\partial f$ is Attouch-Wets proto-differentiable at $\bar{x}$ relative to $\bar{v}$ with $f_{\bar{x}, v}^{\prime \prime}$ primal-lower-nice and

$$
\partial\left((1 / 2) f_{\bar{x}, \bar{v}}^{\prime \prime a w}\right)(\xi)=(\partial f)_{\bar{x}, v}^{\prime a w}(\xi) .
$$

Proof. As in the proof of Theorem 3.8, we are in a situation where we can use Theorem 4.3. This shows that the limiting function $f_{\bar{x}, v}^{\prime \prime}$ is primal-lower-nice on the whole space (because it is twice positively homogeneous). The proof is quite similar in spirit to that of Theorem 3.8 and we therefore omit the details. Needless to say that the key points are that for $\lambda>0$

$$
(\xi, \omega) \in \operatorname{gph} \partial\left(f_{\bar{x}, 0}^{\prime \prime a w}\right) \text { if and only if }(\lambda \xi, \lambda \omega) \in \operatorname{gph} \partial\left(f_{\bar{x}, 0}^{\prime \prime a w}\right)
$$

and that

$$
(\xi, \omega) \in \operatorname{gph} \partial\left(\phi_{t}\right) \text { if and only if }(\lambda \xi, \lambda \omega) \in \operatorname{gph} \partial\left(\phi_{(t / \lambda)}\right) .
$$

\section{REFERENCES}

1. H. Attouch, Variational convergence for functions and operators, Pitman, 1984.

2. H. Attouch, and R. J.-B. Wets, Epigraphical analysis, Analyse Non Linéaire 6 (1989), 73-100.

3. H. Attouch, J.L. Ndoutoume, and M. Thera, Epigraphical convergence of functions and convergence of their derivatives in Banach spaces, Exp. No. 9, Sem. Anal. Convexe (Montpellier) 20 (1990), 9.1-9.45.

4. H. Attouch, R. Lucchetti and R. J.-B. Wets, The topology of the $\rho$-Hausdorff distance, Ann. Mat. Pura Appl. 160 (1991), 303-320.

5. H. Attouch and G. Beer, On the convergence of subdifferentials of.convex functions, Arch. Math. 60 (1993), 389-400.

6. H. Attouch, and R. J.-B. Wets, Quantitative stability of variational systems: I. The epigraphical distance, Trans. Amer. Math. Soc. 328 (1991), 695-729.

7. J. P. Aubin and H. Frankowska, Set-valued analysis, Birkhäuser, 1990.

8. D. Azé and J.P. Penot, Operations on convergent families of sets and functions, Optimization 21 (1990), 521-534. 
9. G. Beer and R. Lucchetti, The epi-distance topology: continuity and stability results with applications to convex optimization problems, Math. Oper. Res. 17 (1992), 715-726.

10. G. Beer and R. Lucchetti, Convex optimization and the epi-distance topology, Trans. Amer. Math. Soc. 327 (1991), 795-813.

11. J.M. Borwein and J.R. Gilles, The proximal normal formula in Banach space, Trans. Amer. Math. Soc. 302 (1987), 371-381.

12. J.M. Borwein and D. Preiss, $A$ smooth variational principle with applications to subdifferentials and to differentiability of convex functions, Trans. Amer. Math. Soc. 303 (1987), 517-527.

13. F.H. Clarke, Optimization and nonsmooth analysis, Centre de Recherches Mathématiques, Université de Montréal (C.P. 6128 "A”, Montrál, Québec, Canada, H3C 3J7), 1989.

14. __ Methods of dynamic and nonsmooth optimization, CBMS-NSF Regional Conference Series in Applied Mathematics, no. 57, 1989.

15. R. Cominetti and R. Correa, A generalized second order derivative in nonsmooth optimization, SIAM J. Control Optim. 28 (1990), 789-809.

16. R. Cominetti, On pseudo-differentiability, Trans. Amer. Math. Soc. 324 (1991), 843-865.

17. R. Correa, A. Joffre and L. Thibault, Characterization of lower semicontinuous convex functions, Proc. Amer. Math. Soc. 116 (1992), 61-72.

18. C. Do, Generalized second derivatives of convex functions in reflexive Banach spaces, Trans. Amer. Math. Soc. 334 (1992), 281-301.

19. A. Levy, Second-order variational analysis with applications to sensitivity in optimization, Ph.D. Thesis, University of Washington, 1994.

20. P.D. Loewen, The proximal subgradient formula in Banach space, Canad. Math. Bull. 31 (1988), 353-361.

21. J.P. Penot, On the convergence of subdifferentials of convex functions, Nonlinear Anal. Th. Meth. Appl. 21 (1993), 87-101.

22. R.A. Poliquin, Proto-differentiation of subgradient set-valued mappings, Canad. J. Math. $\mathbf{4 2}$ (1990), 520-532.

23. 17 (1991), 385-398.

24. _ An extension of Attouch's Theorem and its application to second-order epi-differentiation of convexly composite functions, Trans. Amer. Math. Soc. 332 (1992), 861-874.

25. R.A. Poliquin and R.T. Rockafellar, Amenable functions in optimization, Nonsmooth Optimization Methods and Applications (F. Giannessi, ed.), Gordon \& Breach, Philadelphia, PA, 1992, pp. 338-353.

26. __ A calculus of epi-derivatives applicable to optimization, Canad. J. Math. 45 (1993), 879-896.

27. __ Proto-derivative formulas for basic subgradient mappings in mathematical programming, Set-Valued Analysis 2 (1994), 275-290.

28. R.A. Poliquin, J.Vanderwerff and V. Zizler, Convex composite representation of lower semicontinuous functions and renormings, C.R. Acad. Sci. Paris Sér. I 317 (1993), 545-549.

29. R.T. Rockafellar, Convex analysis, Princeton Univ. Press, Princeton, NJ, 1970.

30. __ Proximal subgradients, marginal values, and augmented Lagrangians in nonconvex optimization, Math. Oper. Res. 6 (1981), 424-436.

31. _ Favorable classes of Lipschitz-continuous functions in subgradient optimization, Progress in Nondifferentiable Optimization (E.Nurminski, ed.), IIASA Collaborative Proceedings Series, International Institute of Applied Systems Analysis, Laxenburg, Austria, 1982, pp. 125-144.

32. - First- and second-order epi-differentiability in nonlinear programming, Trans. Amer. Math. Soc. 307 (1988), 75-107.

33. __ Proto-differentiability of set-valued mappings and its applications in optimization, Analyse Non Linéaire (H. Attouch et al., eds.), Gauthier-Villars, Paris, 1989, pp. 449-482. 
34. __ Perturbation of generalized Kuhn-Tucker points in finite dimensional optimization, Nonsmooth Optimization and Related Topics (F.H. Clarke et al., eds.), Plenum Press, 1989, pp. 393-402.

35. - Second-order optimality conditions in nonlinear programming obtained by way of epiderivatives, Math. Oper. Res. 14 (1989), 462-484.

36. - Generalized second derivatives of convex functions and saddle functions, Trans. Amer. Math. Soc. 320 (1990), 810-822.

37. _ Nonsmooth analysis and parametric optimization, Methods of Nonconvex Analysis (A. Cellina, ed.), Lecture Notes in Math., vol. 1446, Springer-Verlag, 1990, pp. 137-151.

38. R.T. Rockafellar and R. J.-B. Wets, Variational analysis (to appear).

39. L. Thibault and D. Zagrodny, Integration of subdifferentials of lower semicontinuous functions, J. Math. Anal. Appl (to appear).

40. T. Zolezzi, Convergence of generalized gradients, preprint.

Department of Mathematics, Bowdoin College, Brunswick, Maine 04011

E-mail address: alevy@kodiak. bowdoin.edu

Department of Mathematical Sciences, University of Alberta, Edmonton, Alberta, Canada T6G 2G1

E-mail address: rene@fenchel.math.ualberta.ca

Département des Sciences, Université Montpellier II, Mathematiques, Place Eugène Bataillon, 34095 Montpellier Cedex 5, France

E-mail address: thibault@math. univ-montp2.fr 